\title{
Flat inputs: theory and applications
}

\author{
Florentina Nicolau*, Witold Respondek $\stackrel{\dagger}{\text { and Jean-Pierre Barbot }}{ }^{\ddagger}$
}

\begin{abstract}
In this paper, we study the problem of constructing flat inputs for multi-output dynamical systems. The notion of flat inputs has been introduced by Waldherr and Zeitz in [31, 32] and can be seen as dual to that of flat outputs. In the single-output case, a flat input can be constructed if and only if the original dynamical system together with its output is observable. In the multi-output case, the observability is not necessary for the existence of flat inputs. The observable case has been treated in [32, where a system of linear algebraic equations has been proposed in order to determine the control vector fields associated to the flat inputs. The goal of this paper is to treat the unobservable case for multi-output dynamical systems. We start by discussing the case when the dynamical system together with the given output is observable and we present a generalization of the results of [32] by relating them with the notion of minimal differential weight. Then we give our main results. We consider the unobservable case for which locally, around any point of an open and dense subset of $\mathbb{R}^{n}$, we construct control vector fields $g_{1}, \ldots, g_{m}$ such that the associated control system is flat (where $n$ and $m$ denote, resp., the state and the output dimensions). Finally, we explain how our results can be applied to private communication.
\end{abstract}

Keywords: Flat inputs, flatness, observed dynamical systems, constructing control vector fields, private communication.

\section{Introduction}

Consider the following nonlinear observed dynamics:

$$
\Sigma: \dot{x}=f(x), \quad y=h(x),
$$

where $x$ is the state defined on $\mathbb{R}^{n}$ and $y \in \mathbb{R}^{m}$ are the measurements, assumed independent everywhere. The vector field $f$ and the functions $h_{i}, 1 \leq i \leq m$, are supposed smooth (the word smooth will always mean $\mathcal{C}^{\infty}$-smooth). The problem that we are studying in this paper is to find control vector fields $g_{1}, \ldots, g_{m}$ (or equivalently, to place the actuators or

*QUARTZ EA7393 Laboratory, ENSEA, 6 Avenue du Ponceau, 95014 Cergy-Pontoise, France. Research partially supported by the Natural Science Foundation of China (61573192). florentina.nicolau@ensea.fr

${ }^{\dagger}$ Normandie Université, INSA de Rouen, Laboratoire de Mathématiques EA 3226 - FR CNRS 3335, 76801 Saint-Etienne-du-Rouvray, France. witold.respondek@insa-rouen.fr

${ }_{\ddagger}^{\ddagger}$ QUARTZ EA7393 Laboratory, ENSEA, 6 Avenue du Ponceau, 95014 Cergy-Pontoise, France, barbot@ensea.fr 
the inputs) such that the control-affine system $\Sigma_{c}: \dot{x}=f(x)+\sum_{i=1}^{m} g_{i}(x) u_{i}$, associated to $\Sigma$, is flat with the original measurements $\left(h_{1}, \ldots, h_{m}\right)$ being a flat output. The notion of flatness was introduced in control theory in the 1990's, by Fliess, Lévine, Martin and Rouchon [4, 5], see also [12, 13, 19, 26], and has attracted a lot of attention because of its multiple applications in the problems of constructive controllability and motion planning (see [17, 20] and references therein). Flat systems form a class of control systems whose all controls and state trajectories can be parametrized by $m$ functions and their time-derivatives, $m$ being the number of controls. More precisely, the control system $\dot{x}=f(x)+\sum_{i=1}^{m} g_{i}(x) u_{i}$, where $x \in \mathbb{R}^{n}, u \in \mathbb{R}^{m}$, is flat if we can find $m$ functions $\varphi_{i}\left(x, u, \ldots, u^{(q)}\right)$ such that

$$
x=\gamma\left(\varphi, \ldots, \varphi^{(s-1)}\right) \text { and } u=\delta\left(\varphi, \ldots, \varphi^{(s)}\right),
$$

for certain integers $q$ and $s$, where $\varphi=\left(\varphi_{1}, \ldots, \varphi_{m}\right)$ is called a flat output. The construction of a flat output $h=\varphi$ can be seen as a problem of sensor placement in order to achieve flatness of the resulting input-state-output system. Dual to this, one can consider the problem of actuator placement, i.e., given $(f, h)$, find control vector fields $g_{1}, \ldots, g_{m}$, in order to achieve the same property. This dual problem has been introduced by Waldherr and Zeitz [31, 32] who call inputs $u_{1}, \ldots, u_{m}$ multiplying, resp., $g_{1}, \ldots, g_{m}$, as flat inputs (which are objects dual to flat outputs).

In the single-output case, a flat input can be constructed if and only if the system $\Sigma$ together with its output $h$ is observable; moreover, the control vector field associated to the flat input can be computed from a system of linear algebraic equations, see [31]. In the multi-output case, the observability is not necessary for the existence of flat inputs. The observable case has been discussed in [32] and the control vector fields (the number of them is that of outputs) associated to the flat inputs can be determined in a similar way as for the SISO case. Another approach in the observable case, based on the notion of unimodularity, has been recently proposed in [6], together with an algorithm for constructing flat inputs and it is then shown that in the observable case, the integrability condition is always satisfied. The goal of this paper is to treat the unobservable multi-output case (the two-output case has been solved by the authors in [22]). It is crucial to distinguish the observability (or unobservability) of controlled systems from observability of uncontrolled ones (recall that for nonlinear systems, the observability property depends on the control [7, 9]). Here we deal with unobservable uncontrolled system that become at least locally observable due to a suitable design of flat inputs.

In this paper, we give a complete solution for a local construction of flat inputs: we show that locally (around any point of an open and dense subset of the state space, where suitable ranks are constant), for any observed dynamical system $(\Sigma, h)$, observable or not, there always exist control vector fields $g_{1}, \ldots, g_{m}$ such that the control-affine system $\Sigma_{c}$ is flat with $h$ being a flat output. Our results can be summarized by the following:

Theorem 1.1. Any observed dynamical system $(\Sigma, h)$ admits flat inputs almost everywhere (that is, on an open and dense subset $\mathcal{X} \subset \mathbb{R}^{n}$ ). Moreover the control vector fields $g_{1}, \ldots, g_{m}$ can be calculated on $\mathcal{X}$ algebraically.

When comparing the problem of existence of flat inputs with that of verifying flatness for control systems, an interesting phenomenon can be noted: contrary to flat control systems that are very rare (the class of flat control systems is of codimension infinity among all control systems), any dynamical system $(\Sigma, h)$ can be rendered flat by adding suitable control vector fields (or equivalently, suitable flat inputs). Our construction is the simplest possible; namely, 
$g_{1}, \ldots, g_{m}$ are the simplest possible control vector fields: they can be chosen constant if $(\Sigma, h)$ is observable and almost all of them (except $\left.g_{1}\right)$ are constant if $(\Sigma, h)$ is not observable (in that case, they are actually in the multi-chained form when restricted to a suitable submanifold). All results use the following three notions introduced in the paper: observability quasi-indices (called OQI's) for the observable case, partial observability quasi-indices (called POQI's) and cascade observability quasi-indices (called COQI's) for the unobservable case. Our main results are based on several normal forms (for which $h$ is a flat output). It is however important to highlight that for the construction of flat inputs, we do not need to transform the system into the normal forms and moreover, algebraic conditions for calculating vector fields $g_{1}, \ldots, g_{m}$ (that render the system flat) are provided.

One of the motivations to construct a flat input for a given output is that with such an input, the tracking problem for that output can be solved with no need to calculate the zero dynamics (see, e.g., [8]), but constructing flat inputs may be useful for other problems as well: in this paper, we explain how it can be applied to private communication.

The paper is organized as follows. In Section 2, we recall the definition of flatness and the notion of differential weight of a flat system. In Section 3 , we discuss (from the point of view of minimal differential weight) the case when the dynamical system $\Sigma$ together with a given output $h$ is observable and we present a generalization of the results of [32]. In Section 4 , we give our main results: we construct flat inputs in the unobservable case. We completely describe the local case (and, as a consequence, we obtain solutions almost everywhere, that is, on an open and dense subset). We illustrate our results by several examples throughout the paper and we explain how it can be applied to private communication in Section 5 . We provide proofs in Section 6 .

\section{Definitions and problem statement}

Consider a nonlinear control system of the form $\Xi: \dot{x}=f(x)+\sum_{i=1}^{m} g_{i}(x) u_{i}$, where $x \in \mathbb{R}^{n}$ and $u \in \mathbb{R}^{m}$.

Definition 2.1. The system $\Xi: \dot{x}=f(x)+\sum_{i=1}^{m} g_{i}(x) u_{i}$ is $x$-flat around $x_{0}$, generically with respect to $u$, if there exist $m$ smooth functions $\varphi_{i}=\varphi_{i}(x), 1 \leq i \leq m$, defined in a neighborhood $\mathcal{O}$ of $x_{0}$, having the following property: there exist an integer $s \geq 0$ and smooth maps $(\gamma, \delta): \widetilde{\mathcal{O}} \times \mathbb{R}^{m(s-1)} \rightarrow \mathbb{R}^{n} \times \mathbb{R}^{m}$ such that

$$
x=\gamma\left(\varphi, \dot{\varphi}, \ldots, \varphi^{(s-1)}\right) \text { and } u=\delta\left(\varphi, \dot{\varphi}, \ldots, \varphi^{(s)}\right)
$$

for any $C^{s-1}$-control $u(t)$ and corresponding trajectory $x(t)$ that satisfy $(x(t), u(t)) \in \widetilde{\mathcal{O}}$, where either $\widetilde{\mathcal{O}}=\mathcal{O} \times \mathbb{R}^{m}$ or $\widetilde{\mathcal{O}}$ is a subset of $\mathcal{O} \times \mathbb{R}^{m}$ such that for any $x \in \mathcal{O}$, the set $\{u:(x, u) \in \widetilde{\mathcal{O}}\}$ is open and dense in $\mathbb{R}^{m}$. The $m$-tuple $\varphi=\left(\varphi_{1}, \ldots, \varphi_{m}\right)$ is called a flat output.

Remark 2.1. It is commonly accepted [5, 17] that flatness is a local and generic property, that is, even if all functions $\varphi_{i}$ depend on $x$ only and are defined globally, desired description (2) is local (valid for trajectories which stay close to the nominal point) and holds out of singular states and singular values of controls. If $\widetilde{\mathcal{O}}=\mathcal{O} \times \mathbb{R}^{m}$, then representation (2) is global with respect to the controls. In the second case, the condition on $\widetilde{\mathcal{O}}$ simply means that for each $x \in \mathcal{O}$, the set of controls $u$ for which representation (2) holds, is open and 
dense in $\mathbb{R}^{m}$ and the pairs $(x, u) \notin \widetilde{\mathcal{O}}$ form singularities of flatness because $(2)$ fails to hold. From now on, when we say that a control system is $x$-flat (or simply flat) at $x_{0}$, we mean that it is $x$-flat at $x_{0}$, generically with respect to $u$ (i.e., satisfies Definition 2.1).

Remark 2.2. There exists a more general notion of flatness for which the functions $\varphi_{i}$ may depend on the control and its successive time-derivatives up to a certain order $q$ (i.e., $\varphi_{i}=\varphi_{i}\left(x, u, \dot{u}, \ldots, u^{(q)}\right)$, as in the introduction). We do not need this general notion since, in our study, all functions $\varphi_{i}$ will depend on the state $x$ only and singularities will depend on $x$ and/or $u$ (but never on derivatives of $u$ ).

The minimal number of derivatives of components $\varphi_{i}$ of a flat output $\varphi$, needed to express $x$ and $u$, is called the differential weight of that flat output and is formalized as follows [21, 28]. By definition, for any flat output $\varphi$ of $\Xi$ there exist integers $s_{1}, \ldots, s_{m}$ such that

$$
x=\gamma\left(\varphi_{1}, \ldots, \varphi_{1}^{\left(s_{1}\right)}, \ldots, \varphi_{m}, \ldots, \varphi_{m}^{\left(s_{m}\right)}\right) \text { and } u=\delta\left(\varphi_{1}, \ldots, \varphi_{1}^{\left(s_{1}\right)}, \ldots, \varphi_{m}, \ldots, \varphi_{m}^{\left(s_{m}\right)}\right)
$$

Moreover, we can choose $\left(s_{1}, \ldots, s_{m}\right), \gamma$ and $\delta$ such that (see [28]) if for any other $m$-tuple $\left(\tilde{s}_{1}, \ldots, \tilde{s}_{m}\right)$ and functions $\tilde{\gamma}$ and $\tilde{\delta}$, we have

$$
x=\tilde{\gamma}\left(\varphi_{1}, \ldots, \varphi_{1}^{\left(\tilde{s}_{1}\right)}, \ldots, \varphi_{m}, \ldots, \varphi_{m}^{\left(\tilde{s}_{m}\right)}\right) \text { and } u=\tilde{\delta}\left(\varphi_{1}, \ldots, \varphi_{1}^{\left(\tilde{s}_{1}\right)}, \ldots, \varphi_{m}, \ldots, \varphi_{m}^{\left(\tilde{s}_{m}\right)}\right),
$$

then $s_{i} \leq \tilde{s}_{i}$, for $1 \leq i \leq m$. We call $\sum_{i=1}^{m}\left(s_{i}+1\right)=m+\sum_{i=1}^{m} s_{i}$ the differential weight of $\varphi$. A flat output of $\Xi$ is called minimal if its differential weight is the lowest among all flat outputs of $\Xi$. The differential weight of a flat systems $\Xi$ is equal to the differential weight of a minimal flat output, and is at least $n+m$, since we have to express $n$ states and $m$ independent controls and in order to do that, we need at least $n+m$ derivatives (taking into account also those of order zero).

Flatness is a property of the state-space dynamics $\dot{x}=f(x)+\sum_{i=1}^{m} g_{i}(x) u_{i}$ of a control system. It can also be described as a property of the input-state-output map for a dummy output $y$. In fact, $x$-flatness is equivalent to the existence of an $\mathbb{R}^{m}$-valued dummy output $y=\varphi(x)$ that renders $\dot{x}=f(x)+\sum_{i=1}^{m} g_{i}(x) u_{i}$ observable [10, 15, 33] and left-invertible [27]. Indeed, expressing the state as $x=\gamma\left(\varphi, \dot{\varphi}, \ldots, \varphi^{(s-1)}\right)$ and the control as $u=\delta\left(\varphi, \dot{\varphi}, \ldots, \varphi^{(s)}\right)$ corresponds, resp., to observability and left invertibility.

Consider the dynamical system $\Sigma$ whose state $x \in \mathbb{R}^{n}$ (all results of the paper also hold if $x$ is defined on an open subset of $\mathbb{R}^{n}$ or, more generally, on an $n$-dimensional manifold) together with the output $y=h(x) \in \mathbb{R}^{m}$, given by (1). In order to emphasize the fact that the system is observed, we will use the notation $(\Sigma, h)$. When we say that a dynamical system $\Sigma$ is observed, this does not mean that $\Sigma$ is necessarily observable with respect to the output $h$.

The problem that we are studying in this paper is the construction of control vector fields $g_{1}, \ldots, g_{m}$ (whose inputs $u_{1}, \ldots, u_{m}$ are called flat inputs) such that the control-affine system $\Sigma_{c}$ associated to $\Sigma$ and given by

$$
\Sigma_{c}: \dot{x}=f(x)+\sum_{i=1}^{m} g_{i}(x) u_{i},
$$

is $x$-flat with respect to the the original output $\left(h_{1}, \ldots, h_{m}\right)$. In that case, we will say that the pair $\left(\Sigma_{c}, h\right)$ is $x$-flat. As we have already noticed, flatness is closely related to observability. Thus for the problem of constructing flat inputs, it is natural to start by checking 
observability of $\Sigma$ with respect to $h$. We denote by $\mathcal{H}(x)$ the observability codistribution (see, e.g., [10])

$$
\mathcal{H}(x)=\operatorname{span}\left\{\mathrm{d} L_{f}^{j-1} h_{i}(x), j \geq 1,1 \leq i \leq m\right\}
$$

associated with the output $h$. We say that $(\Sigma, h)$ satisfies the observability rank condition [10] if $\operatorname{dim} \mathcal{H}(x)=n$. In our study, we will distinguish the observable case $\operatorname{dim} \mathcal{H}(x)=n$ and the unobservable case $\operatorname{dim} \mathcal{H}(x)=k<n$.

\section{Observable case}

For the construction of flat inputs in the observable case, we need the following definition.

Definition 3.1. The observed system $(\Sigma, h)$ is said to have observability quasi-indices $\left(\rho_{1}, \ldots, \rho_{m}\right)$ at $x_{0} \in \mathbb{R}^{n}$, called OQI's, if

$$
\sum_{i=1}^{m} \rho_{i}=n \quad \text { and } \quad \operatorname{span}\left\{\mathrm{d} L_{f}^{j-1} h_{i}\left(x_{0}\right), 1 \leq j \leq \rho_{i}, 1 \leq i \leq m\right\}=\mathbb{R}^{n} .
$$

If, moreover, the quasi-indices satisfy $\rho_{1} \geq \rho_{2} \geq \ldots \geq \rho_{m}$, then the smallest, in the lexicographic ordering, quasi-indices are called observability indices.

For nonlinear systems, the notion of observability indices goes back to [15], see also [33, 34], while the notion of OQI's is used in [32] under the name of (non-unique) observability indices. For linear systems, an analogous notion was proposed under the name of pseudoobservability indices in [2]. The existence of OQI's, and thus of observability indices, at $x_{0}$ implies observability of $(\Sigma, h)$. More precisely, we can distinguish points in a neighborhood of $x_{0}$ with the help of exactly $\rho_{1}+\ldots+\rho_{m}=n$ derivatives of the output components (actually, using $\rho_{i}$ time-derivatives of $\left.y_{i}(t)=h_{i}(x(t))\right)$. Conversely, if $(\Sigma, h)$ satisfies $\operatorname{dim} \mathcal{H}(x)=n$ for any $x$ in $\mathbb{R}^{n}$, i.e., is locally observable everywhere on $\mathbb{R}^{n}$ (see [10], for various concepts of nonlinear observability), then it possesses OQI's on an open and dense subset $\mathcal{X}^{O} \in \mathbb{R}^{n}$ (see Lemma 4.1 in Section 4 and its proof in Section 6) and thus, although it is locally observable everywhere, fails to have OQI's around singular points of $\mathbb{R}^{n} \backslash \mathcal{X}^{O}$. Contrary to the observability indices (which are unique), the OQI's $\left(\rho_{1}, \ldots, \rho_{m}\right)$ are not unique and we do not suppose any order relation between them. Since OQI's may depend on a point, we say that quasi-indices $\left(\rho_{1}, \ldots, \rho_{m}\right)$ are uniform in a subset $X$ of $\mathbb{R}^{n}$ if $\left(\rho_{1}, \ldots, \rho_{m}\right)$ form quasi-indices at any $x_{0} \in X$. We will keep denoting by $\mathcal{X}$ (sometimes with suitable indices) open and dense subsets of $\mathbb{R}^{n}$ and by $X$ just open subsets of $\mathbb{R}^{n}$.

Example 3.1. Consider the following dynamics

$$
\begin{array}{ll}
\dot{x}_{1}=x_{2}+x_{3} & \dot{x}_{3}=x_{4} \\
\dot{x}_{2}=0 & \dot{x}_{4}=0
\end{array}
$$

with the outputs $y_{1}=x_{1}$ and $y_{2}=x_{3}$. A straightforward calculation shows that both couples $\left(\rho_{1}, \rho_{2}\right)=(2,2)$ and $\left(\rho_{1}, \rho_{2}\right)=(3,1)$ are (uniform) OQI's on $\mathbb{R}^{4}$ (but only the first one corresponds to the observability indices). 
In Example 4.1, we illustrate how for a given system different choices of OQI's may exist on different domains.

The following theorem states that the observed system $(\Sigma, h)$ can be made flat of differential weight $n+m$ (which is the minimal possible) if and only if it admits OQI's. Moreover, Proposition 3.1 gives a system of algebraic equations, whose terms are calculated as Lie derivatives of the outputs, to be solved in order to construct control vector fields (and thus flat inputs). Proofs of Theorem 3.1 and of all results of this section are given in Section 6 .

Theorem 3.1. Consider the observed system $(\Sigma, h)$ around $x_{0} \in \mathbb{R}^{n}$. The following conditions are equivalent:

$(\mathcal{O} 1)$ There exist OQI's $\left(\rho_{1}, \ldots, \rho_{m}\right)$ around $x_{0}$;

(O2) There exist vector fields $g_{1}, \ldots, g_{m}$ such that the system $\left(\Sigma_{c}, h\right)$ is $x$-flat at $x_{0}$ of differential weight $n+m$, with $h$ being a minimal flat output, that is, equivalently, the system $\Sigma_{c}$ is static feedback linearizable with $h_{1}, \ldots, h_{m}$ being linearizing outputs.

Moreover, if $\operatorname{dim} \mathcal{H}(x)=n$, for any $x \in \mathbb{R}^{n}$, then $O Q I^{\prime}$ s exist for any $x_{0} \in \mathcal{X}^{O}$, with $\mathcal{X}^{O}$ an open and dense subset of $\mathbb{R}^{n}$, and thus $g_{1}, \ldots, g_{m}$ of (O) exist on $\mathcal{X}^{O}$.

A constructive procedure to find flat inputs is given as follows (where the value of a differential form $\omega=\sum_{i=1}^{n} \omega_{i}(x) \mathrm{d} x_{i}$ on a vector field $v=\sum_{i=1}^{n} v_{i}(x) \frac{\partial}{\partial x_{i}}$ is $\langle\omega, v\rangle=$ $\left.\sum_{i=1}^{n} \omega_{i}(x) v_{i}(x)\right)$.

Proposition 3.1. Consider the observed system $(\Sigma, h)$ around $x_{0} \in \mathbb{R}^{n}$ and assume that condition $(\mathcal{O} 1)$ of Theorem 3.1 holds. Then all choices of the vector fields $g_{1}, \ldots, g_{m}$ that meet condition (O2) of Theorem 3.1 are given by

(i) $\left\langle\mathrm{d} L_{f}^{q-1} h_{i}, g_{j}\right\rangle=0$ and $\left\langle\mathrm{d} L_{f}^{\rho_{i}-1} h_{i}, g_{j}\right\rangle(x)=D_{i j}(x)$, for $1 \leq i, j \leq m, 1 \leq q \leq \rho_{i}-1$, where $\left(D_{i j}(x)\right)$ is any smooth invertible $m \times m$-matrix

or, equivalently, by

(ii) The distribution $\mathcal{G}=\operatorname{span}\left\{g_{1}, \ldots, g_{m}\right\}$ satisfies

$$
\mathcal{G}^{\perp}=\operatorname{span}\left\{\mathrm{d} L_{f}^{j-1} h_{i}, 1 \leq j \leq \rho_{i}-1,1 \leq i \leq m\right\} .
$$

Moreover, if $\rho=\left(\rho_{1}, \ldots, \rho_{m}\right)$ are uniform OQI's on $X_{\rho}^{O}$ (in particular, on $X_{\rho}^{O}=\mathbb{R}^{n}$ ), then the vector fields $g_{1}, \ldots, g_{m}$ exist globally on $X_{\rho}^{O}$, on which are given by (i), and they yield a global system $\Sigma_{c}$ on $X_{\rho}^{O}$ that is locally flat around any $x \in X_{\rho}^{O}$ with the help of the flat output $\left(\varphi_{1}, \ldots, \varphi_{m}\right)=\left(h_{1}, \ldots, h_{m}\right)$ globally defined on $X_{\rho}^{O}$.

Notice that in the analytic case each set $X_{\rho}^{O}$ is open and dense (so could also be denoted $\mathcal{X}_{\rho}^{O}$ ) but in the $\mathcal{C}^{\infty}$-case the sets $X_{\rho}^{O}$ are, in general, open only. Clearly, in both cases, the open and dense set $\mathcal{X}^{O}$ of Theorem 3.1 is $\mathcal{X}^{O}=\cup X_{\rho}^{O}$, where the union is taken over all OQI's $\rho=\left(\rho_{1}, \ldots, \rho_{m}\right)$, see also Lemma 4.1 and the comments following it. Proposition 3.1 gives the following system of algebraic equations.

Corollary 3.1. In a local coordinate system $x=\left(x_{1}, \ldots, x_{n}\right)$, form the $n \times n$-matrix $H(x)$ whose first $n-m$ rows are the differentials $\mathrm{d} L_{f}^{q} h_{i}(x), 0 \leq q \leq \rho_{i}-2$, and the last $m$ rows are the differentials $\mathrm{d} L_{f}^{\rho_{i}-1} h_{i}(x), 1 \leq i \leq m$. Then the equations of condition (i) of Proposition 3.1 are equivalent to 


$$
H(x) \cdot g(x)=\left(\begin{array}{c}
0 \\
D(x)
\end{array}\right),
$$

where $g(x)=\left(g_{1}(x) \ldots g_{m}(x)\right), 0=0_{(n-m) \times m}$ and $D(x)$ is any smooth invertible $m \times m$ matrix. Under condition (O1) of Theorem 3.1. $H(x)$ is invertible and

$$
g(x)=H^{-1}(x) \cdot\left(\begin{array}{c}
0 \\
D(x)
\end{array}\right) .
$$

Proof. Follows directly from Proposition 3.1 .

The vector fields $g_{1}, \ldots, g_{m}$ lead to a control system $\Sigma_{c}$ that is actually locally static feedback linearizable [11, 14] with $h$ being a linearizing output (or, equivalently, of differential weight $n+m$, see [21] where that equivalence is discussed). The following two results are direct consequences of Theorem 3.1, see Section 6 for their proofs.

Corollary 3.2. If $(\Sigma, h)$ is not locally observable at $x_{0}$ or is locally observable but does not have any $O Q I^{\prime}$ 's and nevertheless admits $g_{1}, \ldots, g_{m}$ such that the system $\left(\Sigma_{c}, h\right)$ is $x$-flat at $x_{0}$ with $\varphi=h$ being a minimal flat output at $x_{0}$, then the control system $\Sigma_{c}$, independently of the choice of $g_{1}, \ldots, g_{m}$, is never static feedback linearizable around $x_{0}$.

Corollary 3.3. Assume that $(\Sigma, h)$ is a linear system of the form $\Sigma: \dot{x}=A x, y=$ $h(x)=C x$. There exist constant vector fields $g_{1}=b_{1}, \ldots, g_{m}=b_{m}$ such that the system $\Sigma_{c}: \dot{x}=A x+\sum_{i=1}^{m} b_{i} u_{i}$ is $x$-flat at $x_{0}$ with flat output $\varphi=y=C x$ if and only if the pair $(A, C)$ is observable.

According to Corollary 3.3 , if the original dynamical system is linear and if we want to obtain a flat control system $\Sigma_{c}$ that is also linear, i.e., $\Sigma_{c}$ is $\dot{x}=A x+\sum_{i=1}^{m} b_{i} u_{i}$, then flat inputs can be constructed if and only if $(\Sigma, h)=(A x, C x)$ is observable. Notice also that no condition on the differential weight of $C x$ as a flat output of the flat linear control system $\Sigma_{c}$ is required, so observability of $(A, C)$ is always necessary. If we do not necessarily require to remain within the linear category, flat inputs can (generically) always be constructed following the procedure explained in Section 4 but for the price of loosing linearity.

Remark 3.1 (Comparison with [32]). Theorem 3.1 and Proposition 3.1 generalize the result of 32 according to which if a system has OQI's $\left(\rho_{1}, \ldots, \rho_{m}\right)$ and the vector fields $g_{1}, \ldots, g_{m}$ satisfy (i) with $D_{i j}(x)=\delta_{i j}$, where $\delta_{i j}$ denotes the Kronecker delta, then the control system $\left(\Sigma_{c}, h\right)$ is flat. All other vector fields $g_{i}$ corresponding to all other invertible matrices $\left(D_{i j}(x)\right)$ render the system feedback linearizable and are given up to a transformation $g_{i} \mapsto \sum_{j=1}^{m} D_{i j}(x) g_{j}$. In our result, we prove additionally that such $g_{i}$ 's (and only such) lead to a flat system $\Sigma_{c}$ of differential weight $n+m$, which is minimal and implies that $\Sigma_{c}$ is static feedback linearizable.

The following example illustrates the last statement of Proposition 3.1. Namely, even if we construct a global system $\Sigma_{c}$, the globally defined flat output $\varphi=h$ guarantees local flatness only, since the map $(x, u) \rightarrow\left(h_{1}, \dot{h}_{1}, \ldots, h_{1}^{\left(s_{1}\right)}, \ldots, h_{m}, \dot{h}_{m}, \ldots, h_{m}^{\left(s_{m}\right)}\right)$ need not be globally invertible.

Example 3.2. Consider the following dynamics on $\mathbb{R}^{2}$

$$
\begin{aligned}
& \dot{x}_{1}=f_{1}\left(x_{1}, x_{2}\right) \\
& \dot{x}_{2}=f_{2}\left(x_{1}, x_{2}\right),
\end{aligned}
$$


with the outputs $y_{1}=\mathrm{e}^{x_{1}} \cos x_{2}$ and $y_{2}=\mathrm{e}^{x_{1}} \sin x_{2}$. Independently of $f=\left(f_{1}, f_{2}\right)^{T}$, the above system is locally everywhere observable, with $\left(\rho_{1}, \rho_{2}\right)=(1,1)$ being uniform observability indices on $\mathbb{R}^{2}$. Hence, by Theorem 3.1, the control vector fields $g_{1}$ and $g_{2}$ exist globally on $\mathbb{R}^{2}$ and can be defined by $L_{g_{j}} h_{i}(x)=D_{i j}(x), 1 \leq i, j \leq 2$, where $\left(D_{i j}(x)\right)$ is any smooth invertible $2 \times 2$-matrix. They yield, on $\mathbb{R}^{2}$, the global control system $\Sigma_{c}: \dot{x}=f(x)+u_{1} g_{1}(x)+u_{2} g_{2}(x)$. The flat output $\varphi(x)=h(x)=\left(\mathrm{e}^{x_{1}} \cos x_{2}, \mathrm{e}^{x_{1}} \sin x_{2}\right)$ is globally defined on $\mathbb{R}^{2}$ but the map $(x, u) \rightarrow(h, \dot{h})$ is not globally invertible (because the map $x \rightarrow h(x)=\left(\mathrm{e}^{x_{1}} \cos x_{2}, \mathrm{e}^{x_{1}} \sin x_{2}\right)$ is not). Notice, however, that $\Sigma_{c}$ is globally flat with another flat output $\tilde{\varphi}=\left(x_{1}, x_{2}\right)$. $\triangleleft$

Notice that a given system may posses many different OQI's, and different OQI's $\left(\rho_{1}, \ldots, \rho_{m}\right)$ lead to different control vector fields $g_{1}, \ldots, g_{m}$, not equivalent via $g_{i} \mapsto \sum_{j=1}^{m} \beta_{i j} g_{j}$, and thus to flat control systems $\left(\Sigma_{c}, h\right)$ that are not static feedback equivalent, see Example 3.3 below. In particular, to any choice of OQI's $\left(\rho_{1}, \ldots, \rho_{m}\right)$, there correspond flat inputs $u_{1}, \ldots, u_{m}$ giving (3) with $s_{i}=\rho_{i}$.

Example 3.3. Consider again system (5) of Example 3.1. For the first couple of quasiindices $\left(\rho_{1}, \rho_{2}\right)=(2,2)$, we get $\mathcal{G}^{\perp}=\operatorname{span}\left\{\mathrm{d} x_{1}, \mathrm{~d} x_{3}\right\}$. Thus $\mathcal{G}=\operatorname{span}\left\{\frac{\partial}{\partial x_{2}}, \frac{\partial}{\partial x_{4}}\right\}$ and the associated flat control system for $D_{i j}(x)=\delta_{i j}, 1 \leq i, j \leq 2$, is

$$
\begin{array}{ll}
\dot{x}_{1}=x_{2}+x_{3} & \dot{x}_{3}=x_{4} \\
\dot{x}_{2}=u_{1} & \dot{x}_{4}=u_{2}
\end{array}
$$

with $h=\left(x_{1}, x_{3}\right)$ a minimal flat output of differential weight $n+2=6$.

The second couple of quasi-indices $\left(\rho_{1}, \rho_{2}\right)=(3,1)$ leads to $\mathcal{G}^{\perp}=\operatorname{span}\left\{\mathrm{d} x_{1}, \mathrm{~d} x_{2}+\mathrm{d} x_{3}\right\}$. Thus $\mathcal{G}=\operatorname{span}\left\{\frac{\partial}{\partial x_{4}}, \frac{\partial}{\partial x_{2}}-\frac{\partial}{\partial x_{3}}\right\}$ and the associated flat control system for $D_{i j}(x)=\delta_{i j}$, $1 \leq i, j \leq 2$, is

$$
\begin{array}{ll}
\dot{x}_{1}=x_{2}+x_{3} & \dot{x}_{3}=x_{4}-u_{1} \\
\dot{x}_{2}=u_{1} & \dot{x}_{4}=u_{2}
\end{array}
$$

with $h=\left(x_{1}, x_{3}\right)$ a minimal flat output of differential weight $n+2=6$.

In some practical applications, it may be interesting to use more derivatives of a particular output component to decrease the number of derivatives of another (more sensitive) component. For instance, in the above example, if the output $y_{1}=x_{1}$ contains less measurement noise than $y_{2}=x_{3}$ or if the system were nonlinear, with fewer nonlinear terms in successive time-derivatives of $y_{1}=x_{1}$ than of $y_{2}=x_{3}$, then it is natural to consider the quasi-indices $\left(\rho_{1}, \rho_{2}\right)=(3,1)$. Notice also that both resulting control systems (6) and (7) are static feedback linearizable with $\left(x_{1}, x_{3}\right)$ playing the role of the linearizing output, but they are not static feedback equivalent to each other. This is so because their quasi-indices coincide with their controllability indices. The former being different implies that the latter are different as well and thus the systems cannot be static feedback equivalent.

Finally, notice that in the observable case, even if the proposed construction of $g_{1}, \ldots, g_{m}$ is local around a given $x_{0}$, description (2) for $\left(\Sigma_{c}, h\right)$ is always global with respect to the control, so we never face singularities in the control space. This is to be expected since our solution leads to a static feedback linearizable system (with $h$ being a linearizing output) and the observability (and thus flatness) properties of a linear system do not depend on controls.

To sum up the above results, if a pair $(\Sigma, h)$ is observable and admits OQI's, then there always exist $g_{1}, \ldots, g_{m}$ such that the associated control system $\Sigma_{c}$ is flat with $h$ being a flat output and, moreover, $g_{1}, \ldots, g_{m}$ can be chosen such that $\Sigma_{c}$ is static feedback linearizable and can be calculated via a system of algebraic equations. Our goal is thus to solve the problem of finding flat inputs for the unobservable multi-output case and to generalize the results of [22] treating the two-output case. 


\section{Main results: unobservable case}

Similarly to the definition of OQI's, we introduce the notion of partial observability quasiindices. Recall that to the output $h$, we associated the observability codistribution $\mathcal{H}$ given by (4).

Definition 4.1. The observed system $(\Sigma, h)$ is said to have partial observability quasiindices $\left(\rho_{1}, \ldots, \rho_{m}\right)$ at $x_{0}$, called POQI's, if in a neighborhood of $x_{0}$,

$$
\begin{gathered}
\operatorname{dim} \mathcal{H}(x)=\text { const. }=k<n \text { and } \\
\operatorname{dim} \operatorname{span}\left\{\mathrm{d} L_{f}^{j-1} h_{i}(x), 1 \leq j \leq \rho_{i}, 1 \leq i \leq m\right\}=\sum_{i=1}^{m} \rho_{i}=k .
\end{gathered}
$$

Remark 4.1. According to the above definition, if the system $(\Sigma, h)$ has POQI's at $x_{0}$, then $\operatorname{dim} \mathcal{H}\left(x_{0}\right)=k<n$, i.e., it is unobservable at $x_{0}$ and, in addition, the associated codistribution $\mathcal{H}(x)$ is of constant rank, equal to $k$, in a neighborhood of $x_{0}$. This means that only $k$ directions can be observed (and this is valid around any point of that neighborhood). The above definition is more restrictive than just the lack of observability at a point: we require the system to be nowhere observable on a whole neighborhood of $x_{0}$ and, moreover, its observability defect to be constant on that neighborhood. It turns out that on an open and dense subset of $\mathbb{R}^{n}$, the lack of observability and the existence of POQI's coincide.

Lemma 4.1 (Genericity of constant rank). For any observed dynamics $(\Sigma, h)$ there exists an open and dense subset $\mathcal{X}^{P} \subset \mathbb{R}^{n}$ on which $\operatorname{dim} \mathcal{H}(x)$ is locally constant and on each connected component $X_{c}^{P}$ of $\mathcal{X}^{P}$ where $\operatorname{dim} \mathcal{H}(x)=$ const. $=k$ (the value of $k$ may depend on the component), POQI's exist at any $x_{0} \in X_{c}^{P}$, if $k<n$, or $O Q I$ 's exist at any $x_{0} \in X_{c}^{P}$, if $k=n$ (in which case it is natural to denote $X_{c}^{P}$ by $X_{c}^{O}$ ).

In the analytic case, the dimension $k$ is the same on all components $X_{c}^{P}$ but it may vary from one $X_{c}^{P}$ to another in the $\mathcal{C}^{\infty}$-case (this does not affect our constructions below, that are local, so given on an arbitrary but fixed connected component). On the other hand, different choices $\left(\rho_{1}, \ldots, \rho_{m}\right)$ of POQI's or OQI's may in general be possible in both analytic and $\mathcal{C}^{\infty}$-case and the domain of validity of each choice $\left(\rho_{1}, \ldots, \rho_{m}\right)$ may be different. If $\operatorname{dim} \mathcal{H}(x)=n$, for all $x \in \mathbb{R}^{n}$, it follows that $(\Sigma, h)$ admits OQI's at any $x_{0} \in \mathcal{X}^{P}$ in which case, we denote the open and dense subset $\mathcal{X}^{P}$ by $\mathcal{X}^{O}$. Proofs of Lemma 4.1 and all results of this section are given in Section 6 .

Example 4.1. Consider the following dynamics

$$
\begin{array}{ll}
\dot{x}_{1}=f_{1}\left(x_{2}\right) & \dot{x}_{3}=x_{4} \\
\dot{x}_{2}=0 & \dot{x}_{4}=f_{4}\left(x_{2}\right)
\end{array}
$$

with the outputs $y_{1}=x_{1}$ and $y_{2}=x_{3}$. We consider the following cases:

(a) $f_{1}=x_{2}$ and $f_{4}=x_{2}$. Then both pairs $(2,2)$ and $(1,3)$ form uniform OQI's on $\mathbb{R}^{4}$.

(b) $f_{1}=x_{2}^{3}$ and $f_{4}=x_{2}$. Then the pair $(1,3)$ forms uniform OQI's on $\mathcal{X}_{(1,3)}^{O}=\mathbb{R}^{4}$ and the pair $(2,2)$ forms uniform OQI's on $\mathcal{X}_{(2,2)}^{O}=\left\{x \in \mathbb{R}^{4}: x_{2} \neq 0\right\}$. The pair $(2,2)$ is that constructed in the proof of Lemma 4.1 . 
(c) $f_{1}=x_{2}^{3}$ and $f_{4}=\left(x_{2}-1\right)^{3}$. Then the pair $(1,3)$ forms uniform OQI's on $\mathcal{X}_{(1,3)}^{O}=\{x \in$ $\left.\mathbb{R}^{4}: x_{2} \neq 1\right\}$ and the pair $(2,2)$ forms uniform OQI's on $\mathcal{X}_{(2,2)}^{O}=\left\{x \in \mathbb{R}^{4}: x_{2} \neq 0\right\}$. Both pairs have singularities which differ from one case to the other.

(d) $f_{1}=x_{2}^{3}$ and $f_{4}=\left\{\begin{array}{l}0, x_{2} \leq 0, \\ \exp \left(-\frac{1}{x_{2}^{2}}\right), x_{2}>0 .\end{array}\right.$ Then the pair (1,3) forms uniform OQI's on $X_{(1,3)}^{O}=\left\{x \in \mathbb{R}^{4}: x_{2}>0\right\}$ and the pair $(2,2)$ forms uniform OQI's on $\mathcal{X}_{(2,2)}^{O}=\mathbb{R}^{4}$. The pair $(2,2)$ is that constructed in the proof of Lemma 4.1. so the pair $(1,3)$ exists on a proper subset $X_{(1,3)}^{O}$ of $\mathcal{X}_{(2,2)}^{O}$.

(e) $f_{1}=\left\{\begin{array}{l}0, x_{2} \leq 0, \\ \exp \left(-\frac{1}{x_{2}^{2}}\right), x_{2}>0\end{array}\right.$ and $f_{4}=x_{2}$. Then the pair (1,3) forms uniform OQI's on $\mathcal{X}_{(1,3)}^{O}=\mathbb{R}^{4}$. The pair $(2,2)$ forms uniform OQI's on $X_{(2,2)}^{O}=\left\{x \in \mathbb{R}^{4}: x_{2}>0\right\}$ and the set $\mathcal{X}^{O}=\mathcal{X}^{P}$ constructed in the proof of Lemma 4.1 is $\mathcal{X}^{O}=\left\{x \in \mathbb{R}^{4}: x_{2}<\right.$ $0\} \cup\left\{x \in \mathbb{R}^{4}: x_{2}<0\right\}$ with OQI's given by $(1,3)$ and $(2,2)$ on two connected components, respectively (although OQI's $(1,3)$ exists everywhere).

The five cases above may serve to illustrate different behaviors of POQI's if we add an unobservable part to the system, like $\dot{x}_{5}=0$.

As for the OQI's, there can be many POQI's and different POQI's $\left(\rho_{1}, \ldots, \rho_{m}\right)$ will lead to different control vector fields $g_{1}, \ldots, g_{m}$ and thus to different flat control systems $\left(\Sigma_{c}, h\right)$, as explained in Section 4 .

Our main results are based on the observation that a flat system is observable with respect to its flat output. To start with, we render $(\Sigma, h)$ observable by completing the original output $h$ to a new output $h^{\ell}=\left(h, \psi^{1}, \ldots, \psi^{\ell}\right)$, such that $\left(\Sigma, h^{\ell}\right)$ is locally observable. For $1 \leq s \leq \ell-1$, each $\psi^{s}$ of $h^{\ell}$ is an $(m-1)$-tuple of independent functions and plays the role of an $\mathbb{R}^{m-1}$-valued dummy output and $\psi^{\ell}$ is an $\mathbb{R}^{m^{\prime}-1}$-valued dummy output, with $2 \leq m^{\prime} \leq m$. This justifies the notation

$$
\bar{m}=\left\{\begin{array}{l}
m, \text { for } 1 \leq s \leq \ell-1 \\
m^{\prime}, \text { for } s=\ell
\end{array}\right.
$$

For any $1 \leq s \leq \ell$, we denote $h^{s}=\left(h, \psi^{1}, \ldots, \psi^{s}\right)$. The integer $\ell$ denotes the number of additional dummy outputs $\psi^{s}$ that have to be added to obtain a locally observable $\left(\Sigma, h^{\ell}\right)$. For each $\psi^{s}=\left(\psi_{2}^{s}, \ldots, \psi_{m}^{s}\right), 1 \leq s \leq \ell$, the components $\psi_{i}^{s}$ are numbered between 2 and $\bar{m}$ (that is, $\psi^{s}=\left(\psi_{2}^{s}, \ldots, \psi_{m}^{s}\right)$, for $1 \leq s \leq \ell-1$, and $\psi^{\ell}=\left(\psi_{2}^{\ell}, \ldots, \psi_{m^{\prime}}^{\ell}\right)$ ).

Definition 4.2. The observed system $(\Sigma, h)$ with POQI's $\rho=\left(\rho_{1}, \ldots, \rho_{m}\right)$ at $x_{0}$ is said to have cascade observability quasi-indices at $x_{0}$, called COQI's, if there exist smooth $\mathbb{R}^{\bar{m}-1}$ valued functions $\psi^{1}, \ldots, \psi^{\ell}$ defined in a neighborhood of $x_{0}$, for an integer $\ell \geq 1$, and integers $\nu^{s}=\left(\nu_{2}^{s}, \ldots, \nu_{m}^{s}\right)$, for $1 \leq s \leq \ell$, such that $\left(\rho, \nu^{1}, \ldots, \nu^{s}\right)$ are POQI's of $\left(\Sigma_{c}, h^{s}\right)$, for any $1 \leq s \leq \ell-1$, and are OQI's of $\left(\Sigma_{c}, h^{\ell}\right)$, for $s=\ell$.

The above definition implies that $\sum_{s=1}^{\ell} \sum_{i=2}^{\bar{m}} \nu_{i}^{s}=n-k$. The name COQI's is suggested by the procedure of completing $h$ to $h^{\ell}$ by adding successively dummy outputs $\psi^{1}, \ldots, \psi^{\ell}$. We will explain, see the algorithm in Section 4.1.1, that $\psi_{i}^{s}$ can be suitably chosen among the coordinates $x_{1}, \ldots, x_{n}$ thus proving that COQI's exist on an open and dense $\mathcal{X}^{C} \subset \mathbb{R}^{n}$. Note that, in general, the inclusion $\mathcal{X}^{C} \subset \mathcal{X}^{P}$ is proper (with both sets being open dense). 
The main result of the paper is summarized by the following theorem (then completed by Theorems 4.2, 4.3 and 4.4 that fully solves the problem of constructing flat inputs. It states that for any observed dynamical system $(\Sigma, h)$, observable or not, we can almost everywhere construct flat inputs.

Theorem 4.1. The following conditions hold:

(Flat 1) Any observed dynamical system $(\Sigma, h)$ admits flat inputs on an open and dense subset $\mathcal{X} \subset \mathbb{R}^{n}$. Moreover the control vector fields $g_{1}, \ldots, g_{m}$ can be calculated on $\mathcal{X}$ via differentiation and algebraic operations.

(Flat2) If $(\Sigma, h)$ is observable and possesses OQI's at $x_{0}$, then it admits flat inputs locally around $x_{0}$.

(Flat3) If $(\Sigma, h)$ is not observable but admits COQI's around $x_{0}$, as introduced in Definition 4.2. then $(\Sigma, h)$ admits flat inputs locally around $x_{0}$.

Remark 4.2. The open and dense subset $\mathcal{X}$ of (Flat 1 ) is always $\mathcal{X}=X^{O} \cup X^{C}$, where $X^{O}$ is the set of points where OQI's exist and $X^{C}$ is the set of points where COQI's exist. If $\operatorname{dim} \mathcal{H}(x)=n$, for all $x \in \mathbb{R}^{n}$ (the observable case), then $X^{C}=\emptyset$ and $\mathcal{X}=X^{O}$ is open and dense (see Theorem 3.1) and thus denoted $X^{O}=\mathcal{X}^{O}$. If $\operatorname{dim} \mathcal{H}(x)<n$, for all $x \in \mathbb{R}^{n}$ (the unobservable case), then $X^{O}=\emptyset$ and $\mathcal{X}=X^{C}$ is open and dense (see Theorem 4.2(A1)) and thus denoted $X^{C}=\mathcal{X}^{C}$. In the $\mathcal{C}^{\infty}$-case with no assumption on the dimension of $\mathcal{H}(x)$, it may happen that, although $\mathcal{X}=X^{O} \cup X^{C}$ is open and dense, neither $X^{O}$ nor $X^{C}$ is dense (both are open only).

Statement (Flat 2) is contained in Theorem 3.1, so we do not discuss it here. In the unobservable case, (Flat 3) can be further detailed when working in well chosen coordinates and will be expressed as Theorems 4.2 and 4.3 if the observability defect verifies $n-k \geq m-1$ (resp., as Theorem 4.4 if $n-k<m-1$ ). All theorems are stated with the help of normal forms. It is however important to highlight that for the construction of flat inputs, we do not need to transform the system into the normal forms and, similarly to the observable case, algebraic conditions for computing $g_{1}, \ldots, g_{m}$ for the original system are provided in the following subsections.

From now on, we consider the unobservable case only, so by Lemma 4.1, there exists an open and dense subset $\mathcal{X}^{P}$ of $\mathbb{R}^{n}$ where POQI's $\left(\rho_{1}, \ldots, \rho_{m}\right)$ are well defined. Locally, around $x_{0} \in \mathcal{X}^{P}$, introduce coordinates

$$
w_{i}^{j}=L_{f}^{j-1} h_{i}, \quad 1 \leq j \leq \rho_{i}, 1 \leq i \leq m,
$$

in which $\Sigma$ can be locally transformed into the following observed-unobserved form

$$
\begin{array}{ll}
\dot{w}_{i}^{j}=w_{i}^{j+1}, & 1 \leq j \leq \rho_{i}-1, \\
\dot{w}_{i}^{\rho_{i}}=a_{i}(w), & 1 \leq i \leq m,
\end{array}
$$

$\dot{z}=b(w, z)$,

with $\sum_{i=1}^{m} \rho_{i}=\operatorname{dim} w=k<n$ and $\operatorname{dim} w+\operatorname{dim} z=n$, where $z$ consists of any coordinates completing $w$ to a coordinate system (for instance, $z$ can be taken as well chosen original coordinates $\left.x_{i_{1}}, \ldots, x_{i_{n-k}}\right)$, and $\left(h_{1}, \ldots, h_{m}\right)=\left(w_{1}^{1}, \ldots, w_{m}^{1}\right)$. The $w$-coordinates of the above form are the states observed with the help of the output $h$ and its successive time-derivatives, 


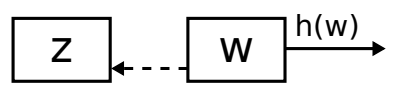

Figure 1: Observed-unobserved subsystems.

and there are $k$ of them. The $z$-coordinates correspond to the unobserved directions, there are $n-k$ (which is the observability defect) of them and they may be affected by $w$. Figure 1 summarizes the above remarks.

Since a flat system is observable (with respect to its flat output and independently of the applied input signal), we have to render the original system $(\Sigma, h)$ observable. For observability we need a link going from the $z$-subsystem into the $w$-subsystem (Figure 2a) but for $\Sigma$ there is no such a link, see the observed-unobserved form (11)-(12) illustrated by Figure $2 \mathrm{~b}$. It follows that we have to create a link assuring observability with the help of the control vector fields (see Figure 2c, where $\Pi$ stands for products $z_{i}^{q+1} u_{1}$ ).

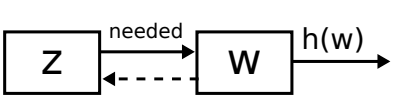

(a) Link needed for observability.

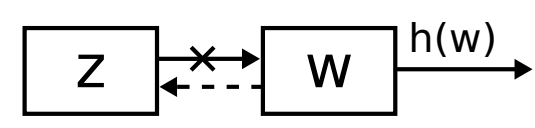

(b) No signal from the $z$-subsystem towards the $w$-subsystem.

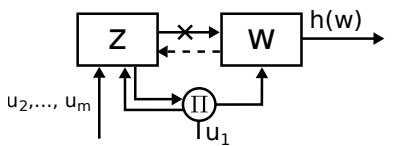

(c) Observability obtained with the $g_{i}$ 's.

Figure 2: Obtaining oservability for $(\Sigma, h)$.

We first give Theorems 4.2 and 4.3 in Section 4.1, which apply to the case $n-k \geq m-1$, and then Theorem 4.4 in Section 4.2 , which deals with $n-k<m-1$.

\subsection{Construction of the control vector fields: the case $n-k \geq m-1$}

Theorem 4.2 presents a normal form NF1 whose construction is performed on a an open and dense subset $\mathcal{X}^{C} \subset \mathbb{R}^{n}$ on which $(\Sigma, h)$ admits COQI's. Recall that COQI's are obtained by completing the original output $h$ to a new output $h^{\ell}=\left(h, \psi^{1}, \ldots, \psi^{\ell}\right)$ such that $\left(\Sigma, h^{\ell}\right)$ is locally observable, see Definition 4.2

Notations of NF1. The integers $\left(\rho, \nu^{1}, \ldots, \nu^{\ell}\right)$ defining NF1, where $\rho=\left(\rho_{1}, \ldots, \rho_{m}\right)$ and $\nu^{s}=\left(\nu_{2}^{s}, \ldots, \nu_{m}^{s}\right)$, for $1 \leq s \leq \ell$, and with $\bar{m}$ defined by $(9)$, are OQI's of $\left(\Sigma, h^{\ell}\right)$, see the algorithm of Section 4.1.1. The variables $z_{i}^{s, q}$ are defined by (18) and form $\ell$ blocks, for $s=1, \ldots, \ell$. In each block, the index $q$ corresponds to successive derivatives and the index $s$ to subsystems. There are $\ell$ subsystems, each of them containing $m-1$ chains $z_{i}^{s}$ (indexed by $i$, for $2 \leq i \leq m$ ), with the exception of the $\ell$-th subsystem, where there can be $m^{\prime}-1<m-1$ chains $z_{i}^{s}$ (indexed by $i$, for $2 \leq i \leq m^{\prime}$ ), i.e., to summarize (using $\bar{m}$-notation), each $z^{s}$-subsystem, for $1 \leq s \leq \ell$, has $\bar{m}-1$ chains. We will also use the following notation

$$
\bar{\ell}= \begin{cases}\ell, & \text { for } 2 \leq i \leq m^{\prime}, \\ \ell-1, & \text { for } m^{\prime}+1 \leq i \leq m\end{cases}
$$

The only nonlinearities of the observed $w$-subsystem are in last $w_{i}^{\rho_{i}}$-components and in the last $z_{i}^{s, q}$-components, with $q=\nu_{i}^{1}, \ldots, \nu_{i}^{\ell}$, of each unobserved $z^{s}$-subsystem, for $s=1, \ldots, \ell$. Only those nonlinear components are modified by the control vector fields $g_{1}, \ldots, g_{m}$. In particular, only to the equations for $\dot{z}_{i}^{\bar{\ell}, q}$, with $q=\nu_{i}^{\bar{\ell}}$, for $2 \leq i \leq \bar{m}$, (that is, for $\dot{z}_{i}^{\ell, q}$, 
with $q=\nu_{i}^{\ell}$, for $2 \leq i \leq m^{\prime}$, and for $\dot{z}_{i}^{\ell-1, q}$, with $q=\nu_{i}^{\ell-1}$, for $m^{\prime}+1 \leq i \leq m$ ), we add, respectively, the term $u_{i}$, while $u_{1}$ affects all other nonlinear components. For $1 \leq s \leq \ell$, denote $\bar{z}^{s}=\left(z_{i}^{j, q}, 1 \leq q \leq \nu_{i}^{j}, 1 \leq j \leq s, 2 \leq i \leq \bar{m}\right)$, i.e., $\bar{z}^{s}$ consists of all components of first $s$ blocks.

Theorem 4.2. Consider $(\Sigma, h)$ and assume that $n-k \geq m-1$. Then:

(A1) There exists $\mathcal{X}^{C} \subset \mathbb{R}^{n}$, open and dense, such that around any $x_{0} \in \mathcal{X}^{C}$, the observed system $(\Sigma, h)$ admits COQI's.

(A2) For any $\psi^{1}, \ldots, \psi^{\ell}$ defining COQI's $\left(\rho, \nu^{1}, \ldots, \nu^{\ell}\right)$ at $x_{0}$ for $(\Sigma, h)$, there exist local coordinates $(w, z)$, with $w$ given by 10 and $z$ consisting of $z_{i}^{s, q}$ given by (18) in Section 4.1 .1 below, bringing $(\Sigma, h)$ into (11) - 12 with the unobserved subsystem (12) of the form:

$$
\begin{gathered}
\dot{z}_{i}^{s, q}= \begin{cases}z_{i}^{s, q+1}, & 1 \leq q \leq \nu_{i}^{s}-1, \\
b_{i}^{s}\left(w, \bar{z}^{s}\right), & q=\nu_{i}^{s}, \\
1 \leq s \leq \ell, 2 \leq i \leq \bar{m}\end{cases}
\end{gathered}
$$

and for this form, we can always locally construct $g_{1}, \ldots, g_{m}$ such that $\left(\Sigma_{c}, h\right)$ is $x$-flat at $x_{0}$ and is given by the following form:

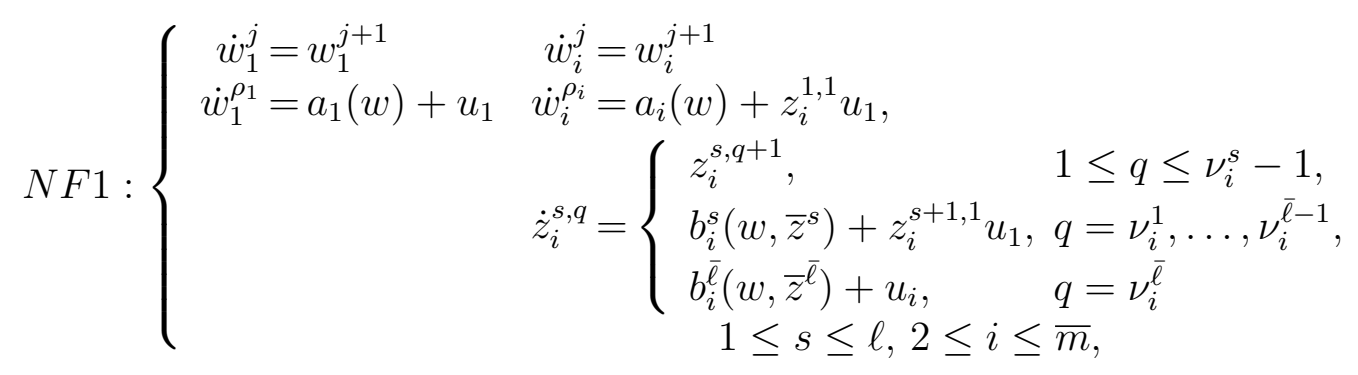

where $1 \leq j \leq \rho_{i}-1$, the integers $\bar{m}$ and $\bar{\ell}$ are given by (9) and (13), respectively, and $h=\left(w_{1}^{1}, \ldots, w_{m}^{1}\right)$ is a flat output of differential weight at least $n+m+\max _{2 \leq i \leq m}\left\{\nu_{i}^{1}+\right.$ $\left.\cdots+\nu_{i}^{\bar{\ell}}\right\}$.

In Section 4.1.1, we present an algorithm constructing, first, the dummy-outputs $\psi^{1}, \ldots, \psi^{\ell}$ on an open and dense subset $\mathcal{X}^{C}$ (and showing the existence of COQI's on $\mathcal{X}^{C}$ ) and, second, the control vector fields $g_{1}, \ldots g_{m}$ of NF1. A proof of Theorem 4.2, based on that algorithm, is given in Section 6.7 .

Theorem 4.2 constructs flat inputs around any $x_{0}$ (equilibrium or not) belonging to an open and dense subset $\mathcal{X}^{C} \subset \mathbb{R}^{n}$. If $x_{0}$ is not an equilibrium point of the original system, i.e., $f\left(x_{0}\right) \neq 0$, then we can locally around $x_{0}$ rectify $f$ and simplify the solution of the problem as described in Theorem 4.3 below. Indeed, first, we render $(\Sigma, h)$ flat around $x_{0}$ provided that it has POQI's at $x_{0}$. Second, the proposed normal form NF2 is obtained by adding to $(\Sigma, h)$ a multi-input chained form for the whole $z$-space (with respect to $z$-variables that rectify the $z$-components of the drift $f)$. Third, the control system $\left(\Sigma_{c}, h\right)$ that we obtain (by adding a multi-input chained form) turns out to be not only flat, which is our primary goal, but the differential weight of $h$ as a flat output of NF2 is the lowest (minimal possible) among all differential weights of $h$ as a flat output of any $\Sigma_{c}$.

Notations of NF2. Recall that $\operatorname{dim} \mathcal{H}(x)=k$ and set 


$$
n-k=p(m-1)+r \quad \text { and } \quad p^{*}=\left\{\begin{array}{l}
p, \text { if } r=0, \\
p+1, \text { if } r>0,
\end{array}\right.
$$

be the Euclidean division of $n-k$ (the observability defect) and $m-1$ (the number of output components being $m$ ). Define

$$
\mu_{i}=\left\{\begin{array}{l}
p, \quad 2 \leq i \leq m-r, \\
p+1, m-r+1 \leq i \leq m,
\end{array} \quad \text { and } \quad b_{i}= \begin{cases}1, & i=2, \\
0, & 3 \leq i \leq m,\end{cases}\right.
$$

that will correspond, respectively, to the lengths and to the last components of the $z_{i}$-chains of the form NF2, (in fact, all last components of $z_{i}$-chains are zero, except that of the $z_{2}$-chain that equals 1 ).

Theorem 4.3. Consider $(\Sigma, h)$ and assume that $n-k \geq m-1$. Then:

(B1) For any $x_{0} \in \mathbb{R}^{n}$ satisfying $f\left(x_{0}\right) \neq 0$ and such that $(\Sigma, h)$ admits POQI's $\left(\rho_{1}, \ldots, \rho_{m}\right)$ at $x_{0}$, there exist local coordinates $(w, z)$ in a neighborhood $X_{0}$ of $x_{0}$, with $w$ given by (10) and $z$ defined explicitly in Section 4.1.2 below, bringing $(\Sigma, h)$ into (11) - (12) with the unobserved subsystem (12) of the form:

$$
\begin{aligned}
\dot{z}_{i}^{q} & =0, \quad 1 \leq q \leq \mu_{i}-1, \\
\dot{z}_{i}^{\mu_{i}} & =b_{i}, \quad 2 \leq i \leq m,
\end{aligned}
$$

where $\mu_{i}$ and $b_{i}$ are given by (16), and for this form, we can always construct vector fields $g_{1}, \ldots, g_{m}$ on $X_{0}$ such that $\left(\Sigma_{c}, h\right)$ is $x$-flat at $x_{0}$ of differential weight $n+m+p^{*}$, and is given by the following form with $h=\left(w_{1}^{1}, \ldots, w_{m}^{1}\right)$ :

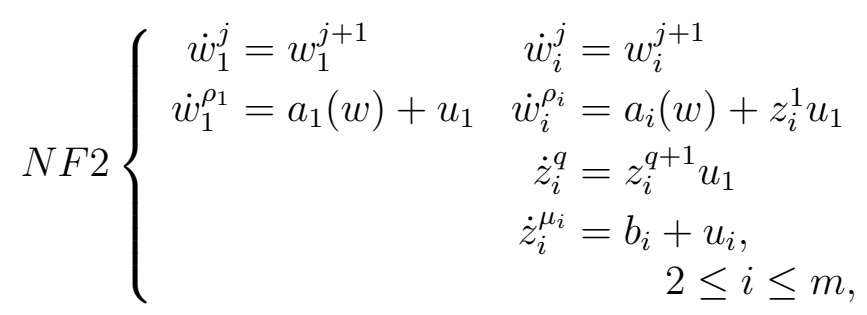

where $1 \leq j \leq \rho_{i}-1,1 \leq q \leq \mu_{i}-1$.

(B2) Let $x_{0} \in \mathbb{R}^{n}$ and assume that $\operatorname{dim} \mathcal{H}(x)=k$ and is constant around $x_{0}$. If there exist $g_{1}, \ldots, g_{m}$ such that $\left(\Sigma_{c}, h\right)$ is $x$-flat at $x_{0}$, then the differential weight of $h$ as a flat output of $\Sigma_{c}$ is at least $n+m+p^{*}$. In particular, the differential weight of $h$ as a flat output of NF2 is the lowest (minimal possible) among all differential weights of $h$ as a flat output of any $\Sigma_{c}$.

An algorithm constructing the $z$-variables and the control vector fields $g_{1}, \ldots, g_{m}$ of normal form NF2 is given in Section 4.1.2, and a proof of Theorem 4.3, based on that algorithm, in Section 6.8.

Remark 4.3 (Singularities in the control space). We would like to emphasize that singularities in the control space are unavoidable (recall that we defined flatness at $x_{0}$ as a generic property with respect to the control $u$, see Definition 2.1). Indeed, if the system $(\Sigma, h)$ is not observable, we add to $f$ the sum $\sum_{i=1}^{m} u_{i} g_{i}$ to render it observable. Now it is clear that if the values of $u$, that form a link between the originally observable and unobservable subsystems, are zero, then the link is broken, the system $\left(\Sigma_{c}, h\right)$ looses its observability and, 
as a consequence, is not flat for those control values. In our forms only the vector field $g_{1}$ (and thus only the input $u_{1}$ ) is used for the link between the observed $w$-subsystem and the unobserved $z$-subsystem, see Figure 2c, while the remaining $u_{2}, \ldots, u_{m}$ affect the unobserved $z$-subsystem only. Hence it is clear that any control $u(t)=\left(u_{1}(t), u_{2}(t), \cdots, u_{m}(t)\right)^{T}$ for which $u_{1}=0$ creates singularities of observability (and thus of flatness).

\subsubsection{Algorithm for constructing the dummy outputs $\psi^{1}, \ldots, \psi^{\ell}$ and the vector field $g_{1}, \ldots, g_{m}$ of normal form NF1}

Consider $(\Sigma, h)$ around any $x_{0} \in \mathbb{R}^{n}$ such that $(\Sigma, h)$ admits POQI's $\left(\rho_{1}, \ldots, \rho_{m}\right)$ at $x_{0}$ (thus, according to Lemma 4.1, on an open and dense subset $\mathcal{X}^{P}$ of $\left.\mathbb{R}^{n}\right)$. Transform it locally into the observed-unobserved form (11)-(12) and denote $x=(w, z)$. We first show the existence of $\psi^{1}, \ldots, \psi^{\ell}$ leading to new coordinates $z$ in which the subsystem $\dot{z}=b(w, z)$ of $(11)-(12)$ takes the form (14) and then for (14) we construct vector fields $g_{1}, \ldots, g_{m}$ yielding normal form NF1. Denote: $\mathcal{X}^{0}=\mathcal{X}^{P}, \mathcal{H}^{0}=\mathcal{H}$, where $\mathcal{H}$ is the observability codistribution given by (4), and $k_{0}=k=\operatorname{dim} \mathcal{H}(x)$.

Choose $m-1$ functions $\psi_{i}^{1}(w, z)$, for $2 \leq i \leq m$, satisfying $\left(\mathrm{d} \psi_{2}^{1} \wedge \ldots \wedge \mathrm{d} \psi_{m}^{1}\right)\left(x_{0}\right) \neq$ $0 \bmod \mathcal{H}^{0}\left(x_{0}\right)$, where, in the $(w, z)$-coordinates, $\mathcal{H}^{0}(x)=\operatorname{span}\left\{\mathrm{d} w_{i}^{j}, 1 \leq i \leq m, 1 \leq\right.$ $\left.j \leq \rho_{i}\right\}$. The functions $\psi_{i}^{1}$ always exist, since $\operatorname{dim} z=p(m-1)+r$, with $p \geq 1$ and $r<m-1$, and can be, for instance, taken as $m-1$ well chosen original coordinates $z_{i_{2}}, \ldots, z_{i_{m}}$. Consider $\psi^{1}=\left(\psi_{2}^{1}, \ldots, \psi_{m}^{1}\right)$ as dummy outputs that complete the original output $h$ to a new output $h^{1}=\left(h, \psi^{1}\right)$ and denote by $\mathcal{H}^{1}$ its associated observability codistribution $\mathcal{H}^{1}(x)=\mathcal{H}^{0}(x)+\operatorname{span}\left\{\mathrm{d} L_{f}^{q-1} \psi_{i}^{1}(x), 2 \leq i \leq m, q \geq 1\right\}$.

There exists an open and dense subset $\mathcal{X}^{1} \subset \mathcal{X}^{0}$ (thus open and dense in $\mathbb{R}^{n}$ as well) such that for any $x_{0} \in \mathcal{X}^{1}$, we can find integers $\nu_{2}^{1}, \ldots, \nu_{m}^{1}$ and a neighborhood $X_{x_{0}}^{1}$ of $x_{0}$ satisfying $\mathcal{H}^{1}(x)=\mathcal{H}^{0}(x)+\operatorname{span}\left\{\mathrm{d} L_{f}^{q-1} \psi_{i}^{1}(x), 2 \leq i \leq m, 1 \leq q \leq \nu_{i}^{1}\right\}$ for $x \in X_{x_{0}}^{1}$, where $\operatorname{dim} \mathcal{H}^{1}(x)=k_{0}+\sum_{i=2}^{m} \nu_{i}^{1}=k_{1}$, for all $x \in X_{x_{0}}^{1}$. Two cases are to be distinguished. Either $k_{1}=n$, for $x \in X_{x_{0}}^{1}$, and then $\left(f, h^{1}\right)$ is observable at $x_{0}$ and $\left(\rho_{1}, \ldots, \rho_{m}, \nu_{2}^{1}, \ldots, \nu_{m}^{1}\right)$ form OQI's at $x_{0}$. Or $k_{1}<n$, for $x \in X_{x_{0}}^{1}$, and then $\left(f, h^{1}\right)$ is not observable at $x_{0}$ and $\left(\rho_{1}, \ldots, \rho_{m}\right.$, $\nu_{2}^{1}, \ldots, \nu_{m}^{1}$ ) are POQI's at $x_{0}$ (of the output $h^{1}$ ). Notice that in both cases, observable and unobservable, the choice of $\nu_{2}^{1}, \ldots, \nu_{m}^{1}$ is, in general, not unique. For $2 \leq i \leq m$, in both cases, introduce coordinates

$$
z_{i}^{1, q}=L_{f}^{q-1} \psi_{i}^{1}, \text { for } 1 \leq q \leq \nu_{i}^{1} .
$$

In the observable case, we set $\ell=1$, and the construction of the $z$-variables ends up, while in the unobservable case, we complete the output $h^{1}$ to a new output $h^{2}=\left(h^{1}, \psi^{2}\right)$ by choosing $m-1$ functions $\psi_{i}^{2}(w, z), 2 \leq i \leq m$, such that $\left(\mathrm{d} \psi_{2}^{2} \wedge \ldots \wedge \mathrm{d} \psi_{m}^{2}\right)\left(x_{0}\right) \neq$ $0 \bmod \mathcal{H}^{1}\left(x_{0}\right)$. Denote by $\mathcal{H}^{2}$ its associated observability codistribution $\mathcal{H}^{2}(x)=\mathcal{H}^{1}(x)+$ span $\left\{\mathrm{d} L_{f}^{q-1} \psi_{i}^{2}(x), 2 \leq i \leq m, q \geq 1\right\}$. There exists an open and dense subset $\mathcal{X}^{2} \subset \mathcal{X}^{1}$ (thus open and dense in $\mathbb{R}^{n}$ as well) such that for any $x_{0} \in \mathcal{X}^{2}$, we can find integers $\nu_{2}^{2}, \ldots, \nu_{m}^{2}$ and a neighborhood $X_{x_{0}}^{2}$ of $x_{0}$ satisfying $\mathcal{H}^{2}(x)=\mathcal{H}^{1}(x)+\operatorname{span}\left\{\mathrm{d} L_{f}^{q-1} \psi_{i}^{2}(x), 2 \leq i \leq m, 1 \leq\right.$ $\left.q \leq \nu_{i}^{2}\right\}$ for $x \in X_{x_{0}}^{2}$, where $\operatorname{dim} \mathcal{H}^{2}(x)=k_{1}+\sum_{i=2}^{m} \nu_{i}^{2}=k_{2}$. For $2 \leq i \leq m$, introduce coordinates

$$
z_{i}^{2, q}=L_{f}^{q-1} \psi_{i}^{2}, \text { for } 1 \leq q \leq \nu_{i}^{2} .
$$

If $k_{2}=n$, we set $\ell=2$, and the construction of the $z$-variables stops, if $k_{2}<n$, we continue. Repeat this process until the system $\left(f, h^{\ell}\right)$, where $h^{\ell}=\left(h, \psi^{1}, \ldots, \psi^{\ell}\right)$, obtained at the $\ell$-th 
step, has OQI's on an open and dense subset $\mathcal{X}^{\ell} \subset \mathcal{X}^{\ell-1}$ (thus open and dense in $\mathbb{R}^{n}$ ). Notice that for any $1 \leq s \leq \ell-1$, we have $\psi^{s}=\left(\psi_{2}^{s}, \ldots, \psi_{m}^{s}\right)$ and $\nu_{i}^{s} \geq 1$, for $2 \leq i \leq m$. Define now the integer $d$ by

$$
d=n-k-\sum_{i=2}^{m} \sum_{s=1}^{\ell-1} \nu_{i}^{s}
$$

and observe that $d$ corresponds to the number of still unobserved directions after performing the penultimate $(\ell-1)$-step, that is, $k+\operatorname{dim} \bar{z}^{\ell-1}=n-d$. For $s=\ell$, we have either $d \geq m-1$ (first case) and then $\psi^{\ell}=\left(\psi_{2}^{\ell}, \ldots, \psi_{m}^{\ell}\right)$ and $\nu_{i}^{\ell} \geq 1$, for $2 \leq i \leq m$ (like in all preceding steps) and we put $m^{\prime}=m$, or $d<m-1$ (second case) and then $\psi^{\ell}=\left(\psi_{2}^{\ell}, \ldots, \psi_{m^{\prime}}^{\ell}\right)$, where $m^{\prime}-1=d$, and $\nu_{i}^{\ell}=\nu_{i}^{\bar{\ell}}=1$ for $2 \leq i \leq m^{\prime}$, see (13) defining $\bar{\ell}$. Recall also the definition of $\bar{m}$ given by $(9)$ and define $\mathcal{X}^{C}=\mathcal{X}^{\ell}$.

Around any point $x_{0} \in \mathcal{X}^{C}=\mathcal{X}^{\ell}$, we introduce $n-k$ coordinates

$$
z_{i}^{s, q}=L_{f}^{q-1} \psi_{i}^{s}, \quad 1 \leq s \leq \ell, 2 \leq i \leq \bar{m}, 1 \leq q \leq \nu_{i}^{s},
$$

that are independent modulo $\mathcal{H}=\operatorname{span}\left\{\mathrm{d} w_{i}^{j}, 1 \leq i \leq m, 1 \leq j \leq \rho_{i}\right\}$, and bring the unobserved subsystem (12) into form (14). Define the control vector fields

$$
g_{1}=\frac{\partial}{\partial w_{1}^{\rho_{1}}}+\sum_{i=2}^{m}\left(z_{i}^{1,1} \frac{\partial}{\partial w_{i}^{\rho_{i}}}+\sum_{s=1}^{\bar{\ell}-1} z_{i}^{s+1,1} \frac{\partial}{\partial z_{i}^{s, \nu_{i}^{s}}}\right) \quad \text { and } \quad g_{i}=\frac{\partial}{\partial z_{i}^{\bar{\ell}, \nu_{i}^{\bar{\ell}}}}, 2 \leq i \leq m \text {. }
$$

In $z_{i}^{s, q}$-coordinates, the vector fields $g_{1}, \ldots, g_{m}$ give normal form NF1 with $\left(h_{1}, \ldots, h_{m}\right)=$ $\left(w_{1}^{1}, \ldots, w_{m}^{1}\right)$, which is clearly $x$-flat at $x_{0}$ with $\left(h_{1}, \ldots, h_{m}\right)$ being a flat output of differential weight at least $n+m+\max _{2 \leq i \leq \bar{m}}\left\{\nu_{i}^{1}+\cdots+\nu_{i}^{\bar{\ell}}\right\}$, see the proof of Theorem 4.2

The above algorithm provides an explicit construction, that uses differentiation and algebraic operations only, of $g_{i}$ 's.

Proposition 4.1. Consider the observed dynamical system $(\Sigma, h)$ around any $x_{0} \in \mathcal{X}^{C}=\mathcal{X}^{\ell}$ and denote $\Psi_{i}^{s, q}=L_{f}^{q-1} \psi_{i}^{s}$, for $1 \leq s \leq \ell, 2 \leq i \leq \bar{m}, 1 \leq q \leq \nu_{i}^{s}$, where the functions $\psi_{i}^{s}$ and the set $\mathcal{X}^{\ell}$ are given by the above algorithm. Denote $\varphi_{i}^{q}=L_{f}^{q-1} h_{i}$, for $1 \leq q \leq \rho_{i}$. Vector fields $g_{1}, \ldots, g_{m}$ such that $\left(\Sigma_{c}, h\right)$ is $x$-flat with $h$ being a flat output can be constructed for $g_{1}$ by

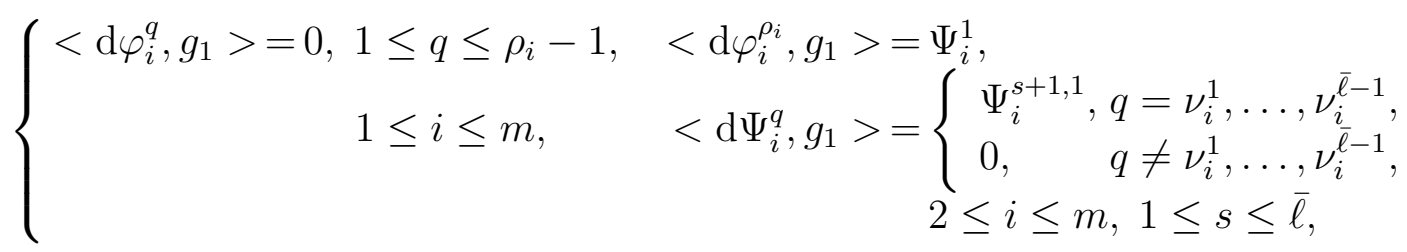

and for $g_{2}, \ldots, g_{m}$ by

$$
\left\{\begin{array}{rl}
<\mathrm{d} \varphi_{i}^{q}, g_{j}>=0,1 \leq q \leq \rho_{i}, \\
1 \leq i \leq m,
\end{array} \quad<\mathrm{d} \Psi_{i}^{s, q}, g_{j}>=\left\{\begin{array}{l}
0, \quad(s, q) \neq\left(\bar{\ell}, \nu_{i}^{\bar{\ell}}\right) \\
\delta_{i j},(s, q)=\left(\bar{\ell}, \nu_{i}^{\bar{\ell}}\right), \\
2 \leq i \leq m, 1 \leq s \leq \bar{\ell}
\end{array}\right.\right.
$$

where $2 \leq j \leq m$, and $\delta_{i j}=1$, if $i=j$, and 0 , otherwise. If all functions $\varphi_{i}^{q}$ and $\Psi_{i}^{s, q}$ are global, i.e., $\overline{\mathcal{X}}^{\ell}=\mathbb{R}^{n}$, then the vector fields $g_{i}$ are defined globally and assure local flatness around any $x_{0} \in \mathbb{R}^{n}$. 
Corollary 4.1. In a local coordinate system $x=\left(x_{1}, \ldots, x_{n}\right)$, form the $n \times n$-matrix $H^{\ell}(x)=\left(\begin{array}{c}H(x) \\ \Psi(x)\end{array}\right)$ whose first $k$ rows $H(x)$ are the differentials $\mathrm{d} \varphi_{i}^{q}(x)=\mathrm{d} L_{f}^{q-1} h_{i}(x)$ and the last $n-k$ rows $\Psi(x)$ are the differentials $\mathrm{d} \Psi_{i}^{s, q}=\mathrm{d} L_{f}^{q-1} \psi_{i}^{s}$, where we use the notations of Proposition 4.1. Then equations (19) and 20) are equivalent to

$$
H^{\ell}(x) \cdot g(x)=D(x),
$$

where $g(x)=\left(g_{1}(x) \ldots g_{m}(x)\right)$, and $D(x)=\left(d_{1}(x) \ldots d_{m}(x)\right)$ with $d_{i}$ denoting suitable terms of the right hand-side of (19) and (20) and both matrices $H^{\ell}(x)$ and $D(x)$ are thus determined by elements of $H(x)$ and $\Psi(x)$. If $x_{0} \in \mathcal{X}^{C}=\mathcal{X}^{\ell}$ (in particular, COQI's exist at $x_{0}$ ), the matrix $H^{\ell}(x)$ is invertible around $x_{0}$ and

$$
g(x)=\left(H^{\ell}\right)^{-1}(x) \cdot D(x) .
$$

Proof. The proofs of Proposition 4.1 and Corollary 4.1 follow directly from the algorithm in Section 4.1.1 for constructing $g_{1}, \ldots, g_{m}$ of NF1.

\subsubsection{Algorithm for constructing the $z$-variables and $g_{1}, \ldots, g_{m}$ of normal form NF2}

Consider $(\Sigma, h)$ around any $x_{0} \in \mathbb{R}^{n}$ satisfying $f\left(x_{0}\right) \neq 0$ and such that $(\Sigma, h)$ admits POQI's $\left(\rho_{1}, \ldots, \rho_{m}\right)$ at $x_{0}$. By $f\left(x_{0}\right) \neq 0$, the first order partial differential equation $L_{f} \psi(x)=$ $\sum_{i=1}^{n} \frac{\partial \psi}{\partial x_{i}} f_{i}=0$ admits $n-1$ solutions independent at $x_{0}$. We choose among them any $n-k-1$ solutions independent modulo $\mathcal{H}=\operatorname{span}\left\{\mathrm{d} L_{f}^{j-1} h_{i}, 1 \leq j \leq \rho_{i}, 1 \leq i \leq m\right\}$ at $x_{0}$ and we label them as $\psi_{i}^{q}, 1 \leq q \leq \mu_{i}$, where $\mu_{i}=p$ for $2 \leq i \leq m-r$ and $\mu_{i}=p+1$ for $m-r+1 \leq i \leq m$, and $(i, q) \neq(2, p)$ (recall that $n-k=p(m-1)+r)$. Choose a solution of $L_{f} \psi(x)=1$, and denote it $\psi_{2}^{p}$, such that the differentials of $\psi_{2}^{p}$ and of the just constructed $\psi_{i}^{q}$ are independent modulo $\mathcal{H}$. Around $x_{0}$, introduce $(w, z)$-coordinates with $w$ given by 10 . and $z_{i}^{q}=\psi_{i}^{q}$, where $2 \leq i \leq m, 1 \leq q \leq \mu_{i}$. For the system $(\Sigma, h)$, whose $w$-subsystem is given by (11) and the $z$-subsystem is represented by (17) in just constructed $z_{i}^{q}$-coordinates, define

$$
g_{1}=\frac{\partial}{\partial w_{1}^{\rho_{1}}}+\sum_{i=2}^{m}\left(z_{i}^{1} \frac{\partial}{\partial w_{i}^{\rho_{i}}}+\sum_{q=1}^{\mu_{i}-1} z_{i}^{q+1} \frac{\partial}{\partial z_{i}^{q}}\right) \quad \text { and } \quad g_{i}=\frac{\partial}{\partial z_{i}^{\mu_{i}}}, \quad 2 \leq i \leq m .
$$

In $\left(w, z_{i}^{q}\right)$-coordinates, the corresponding control system is in the form NF2, with $\left(h_{1}, \ldots, h_{m}\right)=$ $\left(w_{1}^{1}, \ldots, w_{m}^{1}\right)$, which is clearly $x$-flat at $x_{0}$ of differential weight $n+m+p^{*}$ (where $p^{*}=p$, if $r=0$, and $p^{*}=p+1$, if $r>0$, see (15)).

Once independent solutions $\psi_{i}^{q}$ of $L_{f} \psi(x)=0$ and $L_{f} \psi(x)=1$ have been computed, the above algorithm provides an explicit construction of $g_{i}$ 's (that uses differentiation and algebraic operations only in original coordinates).

Proposition 4.2. Consider the dynamical system $(\Sigma, h)$ around any $x_{0} \in \mathbb{R}^{n}$ satisfying $f\left(x_{0}\right) \neq 0$ and such that $(\Sigma, h)$ admits POQI's $\left(\rho_{1}, \ldots, \rho_{m}\right)$ at $x_{0}$. Denote $\varphi_{i}^{q}=L_{f}^{q-1} h_{i}$, for $1 \leq q \leq \rho_{i}$, and compute the functions $\psi_{i}^{q}$, for $1 \leq q \leq \mu_{i}, 2 \leq i \leq m$, of the above algorithm. Vector fields $g_{1}, \ldots, g_{m}$ such that $\left(\Sigma_{c}, h\right)$ is $x$-flat with $h$ being a flat output can be constructed for $g_{1}$ by 


$$
\left\{\begin{array}{cc}
<\mathrm{d} \varphi_{i}^{q}, g_{1}>=0,1 \leq q \leq \rho_{i}-1, & <\mathrm{d} \varphi_{i}^{\rho_{i}}, g_{1}>=\psi_{i}^{1}, \\
<\mathrm{d} \varphi_{1}^{\rho_{1}}, g_{1}>=1, & <\mathrm{d} \psi_{i}^{q}, g_{1}>=\psi_{i}^{q+1}, 1 \leq q \leq \mu_{i}-1, \\
& <\mathrm{d} \psi_{i}^{\mu_{i}}, g_{1}>=0, \\
& 2 \leq i \leq m,
\end{array}\right.
$$

and for $g_{2}, \ldots, g_{m}$ by

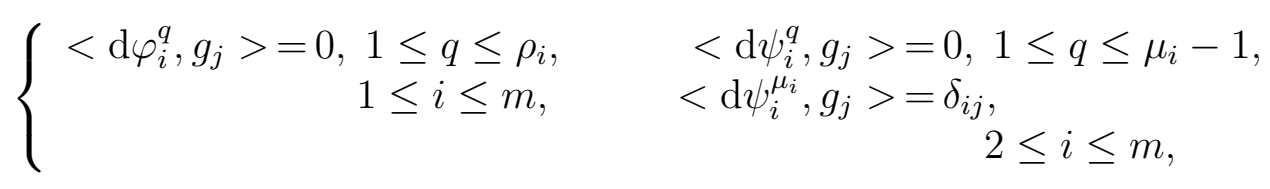

where $2 \leq j \leq m$, and $\delta_{i j}=1$, if $i=j$, and 0 , otherwise. If all functions $\varphi_{i}^{q}$ and $\psi_{i}^{q}$ are global, then the vector fields $g_{i}$ are defined globally and assure local flatness around any $x_{0} \in \mathbb{R}^{n}$.

Proof. Follows directly from the algorithm for constructing $g_{1}, \ldots, g_{m}$ of NF2.

Corollary 4.1 holds also for the computations of $g_{1}(x), \ldots, g_{2}(x)$ of the form NF2, with the $(n-k) \times n$-submatrix $\Psi(x)$ of $H^{\ell}(x)$ and the matrix $D(x)$ being this time defined, respectively, by the differentials $\mathrm{d} \psi_{i}^{q}$, and the right hand-side terms of (24) and (25). In particular, relations (21)-22 remain the same. Notice that now, in order to get the expressions of $\Psi(x)$ and $D(x)$, we first have to compute the solutions $\psi_{i}^{q}$ of $L_{f} \psi(x)=0$ and $L_{f} \psi(x)=1$.

\subsubsection{Discussion of the normal form NF1}

The two extreme cases of the $z$-part of NF1 are $\ell=1$ and $\ell=p$, if $n-k=p(m-1)$, or $\ell=p+1$, if $n-k=p(m-1)+r$, where $1 \leq r \leq m-2$. In the first case, we have (we drop the index $s$ from $z_{i}^{s, q}, \nu_{i}^{s}$ and $b_{i}^{s}$, since it takes only one value, namely $s=1$ )

$$
\begin{aligned}
\dot{z}_{i}^{q} & =z_{i}^{q+1}, \quad 1 \leq q \leq \nu_{i}-1, \\
\dot{z}_{i}^{\nu_{i}} & =b_{i}(w, z)+u_{i}, \quad 2 \leq i \leq m,
\end{aligned}
$$

the $z$-subsystem is formed by $m-1$ almost linear chains (the nonlinearities as well as the inputs appear at the bottom level of each $z_{i}$-chain only). In the second case, we have $\nu_{i}^{s}=1$, for $1 \leq s \leq \ell, 2 \leq i \leq \bar{m}$, where $\bar{m}$ is given by $(9)$ with $m^{\prime}=m$ if $r=0$ and $m^{\prime}=r+1$ if $r>0$, and the $z$-subsystem is given by (we drop the index $q$ from $z_{i}^{s, q}$ since it takes only one value, namely $q=1$ )

$$
\begin{aligned}
& \dot{z}_{i}^{s}=b_{i}^{s}\left(w, \bar{z}^{s}\right)+z_{i}^{s+1} u_{1}, 1 \leq s \leq p, \quad \dot{z}_{i}^{s}=b_{i}^{s}\left(w, \bar{z}^{s}\right)+z_{i}^{s+1} u_{1}, 1 \leq s \leq p-1, \\
& \dot{z}_{i}^{p+1}=b_{i}^{p+1}(w, z)+u_{i}, 2 \leq i \leq r+1, \quad \dot{z}_{i}^{p}=b_{i}^{p}(w, z)+u_{i}, r+2 \leq i \leq m,
\end{aligned}
$$

where the $z_{i}$-chains of length $p+1$ are missing if $r=0$. This case reminds very much form NF2; indeed the vector fields $g_{i}$ are in the multi-chained form and it is due to $g_{1}$ only (the drift does not play any role) that for each $s$, the successive derivatives $L_{g_{1}} z_{i}^{s}=z_{i}^{s+1}$, for $2 \leq i \leq m$, provide $m-1$ new states $z_{i}^{s+1}$ (as many as possible).

While for NF2, the drift is rectified and plays no role in the triangular structure of the $z$-subsystem, for NF1 the drift is not rectified (actually, we cannot rectify it if $x_{0}$ is an equilibrium point). Therefore, we need to identify its triangular structure and to respect it. Any system can always be transformed into NF1 which actually works around any $x_{0}$, equilibrium or not, and in order to compute the $z$-coordinates we do not have to compute any partial differential equation, see the algorithm for the construction of NF1, Proposition 4.1, and 
Corollary 4.1 in Section 4.1.1. For NF1, the linking terms, that is, the nonzero components of $g_{1}$, are the dummy outputs $\psi_{i}^{1}, \ldots, \psi_{i}^{\ell}$ defining, respectively, the variables $z_{i}^{1,1}, \ldots, z_{i}^{\ell, 1}$. Notice that the choice of linking terms $\psi_{i}^{s}$ is far from being unique which may be important in applications.

For fixed $\psi_{i}^{s}$, constructing the $z$-variables (and thus the form NF1) is performed on an open and dense subset $\mathcal{X}^{C}=\mathcal{X}^{\ell} \subset \mathbb{R}^{n}$ (see the algorithm in Section 4.1.1) which we will denote $\mathcal{X}_{\psi}$ to indicate that, indeed, it depends on $\psi$. If it is important to work at a fixed point $x_{0}$ (around which we need COQI's to exist) and if $x_{0} \notin \mathcal{X}_{\psi}$, we can choose another family of dummy outputs $\tilde{\psi}_{i}^{s}$ because, in general, $\mathcal{X}_{\psi} \neq \mathcal{X}_{\tilde{\psi}}$. Choosing different $\tilde{\psi}_{i}^{s}$ 's will lead, in general, to different control vector fields and thus to different flat control systems with different differential weights. Finally, observe that if the system $\Sigma$ is analytic and defined on $\mathbb{R}^{n}$, then there exist functions $\psi^{1}, \ldots, \psi^{\ell}$ globally defined on $\mathbb{R}^{n}$ such that $\left(\Sigma, h^{\ell}\right)$, with $h^{\ell}=\left(h, \psi^{1}, \ldots, \psi^{\ell}\right)$, admits COQI's on an open and dense subset of $\mathbb{R}^{n}$ (that is, the same choice of $\psi^{1}, \ldots, \psi^{\ell}$ works around any $x_{0}$ of that open and dense subset).

For NF1 (resp., NF2), in order to connect the $w$ - and the $z$-subsystems, one linking term for each $w_{i}$-chain is needed and the variables $\left(z_{2}^{1,1}, \ldots, z_{m}^{1,1}\right)$ for NF1 (resp., $\left(z_{2}^{1}, \ldots, z_{m}^{1}\right)$ for NF2) play indeed the role of those linking terms, as $z_{i}^{1,1}$ (resp., $z_{i}^{1}$ ) affects $\dot{w}_{i}^{\rho_{i}}$. The link between the observed $w$-subsystem and the unobserved $z$-subsystem for both NF1 and NF2 as well as the link between the $z^{s}$-blocks is made with the help of the control vector field $g_{1}$ only and the remaining $m-1$ inputs $u_{2}, \ldots, u_{m}$ appear at the bottom level of each $z_{i}$-chain. It follows that the input $u_{1}$ always needs to be differentiated in order to express all states and controls with the help of flat outputs $h_{1}=w_{1}^{1}, \ldots, h_{m}=w_{m}^{1}$ and their derivatives. While for NF2, the control $u_{1}$ is the only one that has to be differentiated, for NF1 other controls among the remaining ones may also need to be differentiated (implying that the differential weight of $h$ is, in general, not minimal, see the proof of Theorem 4.2).

\subsubsection{Discussion of the normal form NF2}

If the observability defect verifies $n-k=p(m-1)$, i.e., $n-k$ is a multiple of $m-1$, then each $z_{i}$-chain is of length $p$, and the control $u_{1}$ needs to be differentiated $p$ times, so the differential weight is $n+m+p$. If $n-k=p(m-1)+r$, with $1 \leq r \leq m-2$, then the $r$ last $z_{i}$-chains are of length $p+1$, the input $u_{1}$ needs to be differentiated $p+1$ times and, hence the differential weight is $n+m+p+1$.

If $f\left(x_{0}\right) \neq 0$, then the drift corresponding to the $z$-variables, that we denote by $b(w, z)$ in (12), can be rectified (even if $b\left(w_{0}, z_{0}\right)=0$ and a non vanishing component of $f$ is in the $w$-part) and, in well chosen $z$-coordinates, we have $b(w, z)=\frac{\partial}{\partial z_{2}^{\mu_{2}}}$ (which is of the form (17)). It follows that there is a significant freedom in constructing $g_{i}$ 's, see the algorithm describing NF2 and Proposition 4.2 in Section 4.1.2. First, the condition $f\left(x_{0}\right) \neq 0$ allows to choose $z_{i}^{q}$ as any independent (modulo $\mathcal{H}$ ) solutions of $L_{f} \psi(x)=0$ and $L_{f} \psi(x)=1$ (for $z_{2}^{p}=\psi_{2}^{p}$ ). Second, there is a full arbitrariness of labelling them as $\psi_{i}^{q}$. In particular $\mu_{i}$ can be taken not as $p$ or $p+1$ but (almost) arbitrarily respecting $\sum_{i=2}^{m} \mu_{i}=n-k$. Our choice is simple (the form of $g_{i}$ 's is the simplest possible) and natural (chosen $\mu_{i}$ give the minimal differential weight) but other choices may also be interesting (in applications, for instance).

Notice that the components of the vector fields $g_{1}, \ldots, g_{m}$ depend on the unobserved (with respect to the original output $h$ ) states $z_{i}^{q}$ only and they are designed to be in the multichained form. This particular form guarantees that the differential weight of $h$ as a flat output of NF2 is the minimal possible (equal to $n+m+p^{*}$ ) among all differential weights of $h$ as a 
flat output of any $\Sigma_{c}$. It is clear that NF2 is not linearizable via invertible static feedback, however, NF2 becomes static static feedback linearizable after pre-integrating $p^{*}$ times the first control $u_{1}$, thus after the application of a dynamical precompensator of dimension $p^{*}$.

\subsection{Construction of the control vector fields: the case $n-k<m-1$}

If the observability defect satisfies $n-k=r<m-1$ (i.e., $p=0$, see (15)), then a result similar to Theorem 4.2 holds (but including it into Theorem 4.2 would significantly complicate the notations) and is given by Theorem 4.4 below.

Theorem 4.4. Consider $(\Sigma, h)$ and assume that $n-k=r<m-1$. Then:

(A1)' There exists $\mathcal{X}^{P} \subset \mathbb{R}^{n}$, open and dense, such that $(\Sigma, h)$ admits POQI's around any point of $\mathcal{X}^{P}$, and at any point at which POQI's exist, COQI's exist as well.

(A2)' Around any point $x_{0}$ of $\mathcal{X}^{P}$, independently of whether $f\left(x_{0}\right)=0$ or $f\left(x_{0}\right) \neq 0$, choose any $z$-coordinates $\left(z_{m^{\prime}+1}^{1}, \ldots, z_{m}^{1}\right)$, where $m^{\prime}=m-r$, completing the $w$-coordinates, given by (10), to a local coordinate system and transform $\Sigma$ into (11)-(12). For that form, the vector fields

$$
\begin{aligned}
g_{1}=\frac{\partial}{\partial w_{1}^{\rho_{1}}}+\sum_{i=m^{\prime}+1}^{m} z_{i}^{1} \frac{\partial}{\partial w_{i}^{\rho_{i}}}, \quad g_{i} & =\frac{\partial}{\partial w_{i}^{\rho_{i}}}, \text { for } 2 \leq i \leq m^{\prime}, \\
g_{i} & =\frac{\partial}{\partial z_{i}^{1}}, \text { for } m^{\prime}+1 \leq i \leq m,
\end{aligned}
$$

yield the system $\left(\Sigma_{c}, h\right)$ that is $x$-flat at $x_{0}$ and given by

$$
N F 3\left\{\begin{array}{rlrl}
\dot{w}_{i}^{j}=w_{i}^{j+1} & \dot{w}_{i}^{j}=w_{i}^{j+1} \\
\dot{w}_{i}^{\rho_{i}}=a_{i}(w)+u_{i}, & \dot{w}_{i}^{\rho_{i}}=a_{i}(w)+z_{i}^{1} u_{1}, \\
1 \leq i \leq m^{\prime}, & \dot{z}_{i}^{1}=b_{i}^{1}(w, z)+u_{i}, \\
& & m^{\prime}+1 \leq i \leq m,
\end{array}\right.
$$

where $b_{i}^{1}(w, z)$ are arbitrary functions of $w$ and $z$, and $h=\left(w_{1}^{1}, \ldots, w_{m}^{1}\right)$ is a flat output of of differential weight $n+m+1$. In particular, if $\mathcal{X}^{P}=\mathbb{R}^{n}$, then $g_{1}, \ldots, g_{m}$ exist locally around any $x_{0} \in \mathbb{R}^{n}$.

(B2)' Let $x_{0} \in \mathbb{R}^{n}$ and assume that $\operatorname{dim} \mathcal{H}(x)=k$ and is constant around $x_{0}$. If there exist $g_{1}, \ldots, g_{m}$ such that $\left(\Sigma_{c}, h\right)$ is $x$-flat at $x_{0}$ then the differential weight of $h$ as a flat output of $\Sigma_{c}$ is at least $n+m+1$. In particular, the $g_{i}$ 's defining NF3 give the lowest (minimal possible) differential weight of $h$ among all choices of $g_{1}, \ldots, g_{m}$.

\subsubsection{Algorithm for constructing $g_{1}, \ldots, g_{m}$ of the normal form NF3}

Consider the dynamical system $(\Sigma, h)$ around any $x_{0}$ such that $(\Sigma, h)$ admits POQI's $\left(\rho_{1}, \ldots, \rho_{m}\right)$ at $x_{0}$ and transform it locally into the observed-unobserved form (11)-(12). Since $\operatorname{dim} z=$ $r<m-1$, it follows that at least $m^{\prime}=m-r$ controls, say $u_{1}, \ldots, u_{m^{\prime}}$, must affect the $w$-subsystem. So we can choose $g_{i}=\frac{\partial}{\partial w_{i}^{\rho_{i}}}$, for $2 \leq i \leq m^{\prime}$ (the control $u_{1}$ will play a special role), and thus modify the corresponding $w$-equations as $\dot{w}_{i}^{\rho_{i}}=a_{i}(w)+u_{i}$, for $2 \leq i \leq m^{\prime}$. The remaining equations for $\dot{w}_{i}^{\rho_{i}}$ have to be linked to the $z$-subsystem to render the whole system observable and we use all $z$-variables, where $z=\left(z_{m^{\prime}+1}^{1}, \ldots, z_{m}^{1}\right)$, as linking terms 
multiplying the control $u_{1}$, that is, we define $g_{1}$ and $g_{i}$, for $m^{\prime}+1 \leq i \leq m$, by (26), to get $\dot{w}_{1}^{\rho_{1}}=a_{i}(w)+u_{1}, \dot{w}_{i}^{\rho_{i}}=a_{i}(w)+z_{i}^{1} u_{1}$ and $\dot{z}_{i}^{1}=b_{i}^{1}(w, z)+u_{i}, m^{\prime}+1 \leq i \leq m$. We thus have obtained the normal form NF3 which is clearly $x$-flat at $x_{0}$, with $\left(h_{1}, \ldots, h_{m}\right)=\left(w_{1}^{1}, \ldots, w_{m}^{1}\right)$ being a flat output of differential weight $n+m+1$.

Like for the form NF1, the above algorithm provides an explicit construction, that uses differentiation and algebraic operations only, of $g_{i}$ 's. The algebraic system giving $g_{1}, \ldots, g_{m}$ for NF3 is very similar to that for NF1 and left to the reader.

\subsubsection{Discussion of the normal form NF3}

If $f\left(x_{0}\right) \neq 0$, the drift $b$ of the unobserved subsystem $\sqrt{12}$ can be rectified and, in well chosen $z$-coordinates, we obtain the normal form NF3 with $b_{m^{\prime}+1}^{1}=1$ and $b_{i}^{1}=0$, for $m^{\prime}+2 \leq i \leq m$. This is the analogue of NF2 in the case $n-k<m-1$. The main difference between NF3 and the forms NF1 and NF2 is, as explained in the above algorithm, that the unobserved $z$-part of NF3 can be affected by at most $r=n-k$ inputs, so the remaining controls necessarily appear in the $w$-equations. As for the case $n-k \geq m-1$, the control vector fields are designed to be in the simplest possible form and only the vector field $g_{1}$ is used to link the observed and the unobserved subsystems although $g_{2}, \ldots, g_{m-r}$ affect the $w$-subsystem of NF3 as well (which is, in the case $n-k \geq m-1$, affected by $g_{1}$ only). This, together with the fact that each $z_{i}$-chain is of length one, guarantee that the differential weight of $h$ as a flat output of NF3 is the minimal possible, which is $n+m+1$ (recall that flatness of differential weight $n+m$ characterizes the observable case $(\Sigma, h)$, see Section 2). Notice also that in the case $n-k=r<m-1$, we are able to construct $g_{1}, \ldots, g_{m}$ around any $x_{0}$ of $\mathcal{X}^{P}$, the open and dense subset of $\mathbb{R}^{n}$ consisting of points at which $(\Sigma, h)$ admits POQI's, while Theorem 4.2 , treating the case $n-k \geq m-1$, enables us to construct $g_{1}, \ldots, g_{m}$ around any $x_{0}$ in an open and dense subset $\mathcal{X}^{C}$ of $\mathcal{X}^{P}$ (but, in general, not equal to $\mathcal{X}^{P}$, see Section 4.1.1). In particular, if POQI's exist around any $x_{0} \in \mathbb{R}^{n}$, then NF3 is established around any $x_{0} \in \mathbb{R}^{n}$ and not only on an open and dense subset like NF1.

Summing up, in all cases, we can always construct (locally, on an open and dense subset of $\mathbb{R}^{n}$ ), control vector fields $g_{1}, \ldots, g_{m}$ such that the associated control system $\left(\Sigma_{c}, h\right)$ is flat and thus, generically, the problem of constructing flat inputs is completely solved.

\subsection{Avoiding singularities in the control space}

If the system $(\Sigma, h)$ is observable at a given $x_{0}$, then for the corresponding flat control system $\left(\Sigma_{c}, h\right)$, constructed by Theorem 3.1, Proposition 3.1, and Corollary 3.1, even if $\varphi_{i}=h_{i}$ yield local flatness only, representing the states and the controls with the help of $\varphi_{i}=h_{i}$ and their derivatives is global with respect to $u$, so we never face singularities in the control space. This is no longer the case if $(\Sigma, h)$ is unobservable and all normal forms NF1, NF2, and NF3 exhibit singularities in the control space. Namely, for all of them, the system ceases to be flat (with $h$ as a flat output) at $u_{1}=0$ (which is a singular control for flatness, see Remark 4.3. If we want to avoid singularities in the control space, we can construct another control system as follows: in all equations of NF1, NF2, and NF3, we replace $u_{1}$ by $\exp \left(u_{1}\right)$, except for the equation $\dot{w}_{1}^{\rho_{1}}=a_{1}(w)+u_{1}$ that we keep unchanged. For instance, for NF1, the equations involving $u_{1}$ become:

$$
\begin{aligned}
\dot{w}_{1}^{\rho_{1}}=a_{1}(w)+u_{1} & \dot{w}_{i}^{\rho_{i}}=a_{i}(w)+z_{i}^{1,1} \exp \left(u_{1}\right) \\
& \dot{z}_{i}^{s, q}=b_{i}^{s}\left(w, \bar{z}^{s}\right)+z_{i}^{s+1,1} \exp \left(u_{1}\right), q=\nu_{i}^{1}, \ldots, \nu_{i}^{\bar{\ell}-1}
\end{aligned}
$$


where $1 \leq s \leq \ell, 2 \leq i \leq \bar{m}$. We have thus constructed a new control system $\Sigma_{c}$ that does no longer display singularities in the control space (representing the state $z$ and the control $u$, with the help of components of $\varphi_{i}=h_{i}$ and their derivatives, is global with respect to $u$ ), but the system is nonlinear with respect to $u_{1}$ (which is the price for avoiding singularities).

\section{Application to private communication}

Since the trendsetting work of [25], it is known that the problem of private communication can be investigated with the help of a unidirectional synchronization of chaotic systems (see also [24] for an observer point of view). The goal of this section (which is based on the preliminary paper [23]) is to explain how constructing flat inputs for a given observed dynamics can be applied to private communication via both, using chaotic models and including the messages into the dynamics (see also [1, for a related approach using the design of nonlinear observers with unknown inputs, and [3] and [30], where invertibility and flatness of switched linear discrete-time systems is applied to private communication).

The general problem can be summarized as follow. Suppose that $m$ confidential messages $u_{1}(t), \ldots, u_{m}(t)$ have to be sent to the receiver. To this aim, we use a transmitter $(\Sigma, h)$ that consists of a dynamical system together with an output

$$
\Sigma: \dot{x}=f(x), y=h(x), \quad x \in \mathbb{R}^{n}, y \in \mathbb{R}^{m} .
$$

In order to transmit messages $\left(u_{1}(t), \ldots, u_{m}(t)\right)$, we will add to $\Sigma$ control vector fields $g_{1}, \ldots, g_{m}$ whose controls are, resp., $u_{1}, \ldots, u_{m}$ (that is, messages to be sent) in such a way that

$$
\Sigma_{c}: \dot{x}=f(x)+\sum_{i=1}^{m} u_{i} g_{i}(x), y=h(x),
$$

is a flat system with $\varphi=\left(h_{1}, \ldots, h_{m}\right)$ being a flat output. Both the sender and receiver know completely the equations of the flat control system $\Sigma_{c}: \dot{x}=f(x)+\sum_{i=1}^{m} u_{i} g_{i}(x)$ and in order to transmit $u(t)=\left(u_{1}(t), \ldots, u_{m}(t)\right)$, the sender calculates the corresponding output signal $y(t)=h(x(u(t), t))$, where $x(u(t), t)$ is the solution corresponding to $u(t)$, and transmits the masked information via the communication multiplexed channel. The receiver receives the signal $y(t)$ and knowing $\Sigma_{c}$ calculates $u(t)$ (recall that $\left(\Sigma_{c}, h\right)$ is flat). The original output $h$ being a flat output of $\left(\Sigma_{c}, h\right)$ is essential to express the confidential message $u(t)$ as function of the masked information $y(t)$ sent to the receiver (we thus study not only a synchronization problem, but also an input reconstruction problem). In order to compute $u(t)$ based on the knowledge of $y(t)$, left invertibility of $\Sigma_{c}$ (which is a weaker property than flatness) would be sufficient but, in that case, the receiver should know not only $y(t)$ but also the initial condition and the internal dynamics of the inverse, see [27]. So the advantage of using the flat system $\Sigma_{c}$ is that the receiver uses the masked information $y(t)$ only. To assure that $y(t)$ is well masked (cannot be decrypted by a third person), we use as $\Sigma$ a chaotic system. To illustrate that general scheme, assume that two messages $u_{1}(t)$ and $u_{2}(t)$ have to be sent to the receiver. We use a transmitter, composed of two independent chaotic systems, a Lorenz circuit $\left(x_{\ell}, y_{\ell}, z_{\ell}\right)$, see, e.g., [18], and a Rössler circuit $\left(x_{r}, y_{r}, z_{r}\right)$, see, e.g., [29], of the form (the notation $(C h)$ referring to the chaotic behavior):

$$
(C h): \begin{cases}\dot{x}_{\ell}=\sigma\left(y_{\ell}-x_{\ell}\right) & \dot{x}_{r}=-y_{r}-z_{r} \\ \dot{y}_{\ell}=-k_{d} x_{\ell} z_{\ell}+r x_{\ell}-y_{\ell} & \dot{y}_{r}=x_{r}+a y_{r} \\ \dot{z}_{\ell}=k_{d} x_{\ell} y_{\ell}-\beta z_{\ell} & \dot{z}_{r}=b+z_{r}\left(x_{r}-c\right),\end{cases}
$$


where the parameters $a, b, c, \beta, \sigma, r$ and $k_{d}$ are constant. As explained above, in order to transmit messages $u_{1}(t)$ and $u_{2}(t)$, we add to $(C h)$ two control vector fields $g_{1}$ and $g_{2}$ whose controls are, resp., $u_{1}$ and $u_{2}$ :

$$
\left(C h_{c}\right): \dot{x}=f(x)+u_{1} g_{1}(x)+u_{2} g_{2}(x), y_{i}=h_{i}(x), 1 \leq i \leq 2,
$$

and the masked information transmitted via the communication multiplexed channel is $\left(y_{1}(t), y_{2}(t)\right)=\left(x_{\ell}(t), z_{\ell}(t)\right)$, where $g_{1}$ and $g_{2}$ are chosen in such a way that $\left(C h_{c}\right)$ is flat with $\varphi=\left(h_{1}, h_{2}\right)=\left(x_{\ell}, z_{\ell}\right)$ being a flat output, and $f$ is the right-hand side of $(C h)$. The chaotic behavior of $(C h)$ (depending on the values of the constant parameters) is crucial and has to be preserved by adding the modification $u_{1} g_{1}(x)+u_{2} g_{2}(x)$ to $(C h)$. It is clear that with $\left(h_{1}, h_{2}\right)=\left(x_{\ell}, z_{\ell}\right)$ only the Lorenz variables can be observed and in the observed-unobserved form (11)-(12), using the new global $w$-coordinates $w_{1}^{1}=x_{\ell}$, $w_{1}^{2}=L_{f} x_{\ell}=\sigma\left(y_{\ell}-x_{\ell}\right), w_{2}^{1}=z_{\ell}$, the Lorenz circuit is equivalently given by: $\dot{w}_{1}^{1}=w_{1}^{2}$, $\dot{w}_{1}^{2}=-\sigma k_{d} w_{1}^{1} w_{2}^{1}+\sigma(r-1) w_{1}^{1}-(\sigma+1) w_{1}^{2}=a_{1}(w)$ and $\dot{w}_{2}^{1}=k_{d} w_{1}^{1}\left(\frac{1}{\sigma} w_{1}^{2}+w_{1}^{1}\right)-\beta w_{2}^{1}=a_{2}(w)$. Notice that here, the unobserved subsystem described by the Rössler circuit is completely independent of the observed Lorenz circuit. Define the linking term $z_{2}^{1}=\psi\left(w, x_{r}, y_{r}, z_{r}\right)$ by $\psi=y_{r}$ and compute its successive time-derivatives (we drop the index $s$ since, as we will see, only one dummy output $\psi=y_{r}$ is necessary to render the system observable, i.e., $s$ takes only one value, namely $s=1)$. We get $L_{f} \psi=x_{r}+a y_{r}$ and $L_{f}^{2} \psi=-\left(y_{r}+z_{r}\right)+a\left(x_{r}+a y_{r}\right)$. It is clear that $\mathrm{d} \psi, \mathrm{d} L_{f} \psi$ and $\mathrm{d} L_{f}^{2} \psi$ are independent everywhere, and $z_{2}^{q}=L_{f}^{q-1} \psi, 1 \leq q \leq 3$, (together with the $w$-coordinates) define a global system of coordinates on $\mathbb{R}^{3} \times \mathbb{R}^{3}$. According to our results, we globally define $g_{1}=\frac{\partial}{\partial w_{1}^{2}}+z_{2}^{1} \frac{\partial}{\partial w_{2}^{1}}$ and $g_{2}=\frac{\partial}{\partial z_{2}^{3}}$ and obtain in $(w, z)$-coordinates a system in NF1-form

$$
N F 1: \begin{cases}\dot{w}_{1}^{1}=w_{1}^{2} & \dot{w}_{2}^{1}=a_{2}(w)+z_{2}^{1} u_{1} \\ \dot{w}_{1}^{2}=a_{1}(w)+u_{1} & \dot{z}_{2}^{1}=z_{2}^{2} \\ & \dot{z}_{2}^{2}=z_{2}^{3} \\ & \dot{z}_{2}^{3}=b_{2}(w, z)+u_{2}\end{cases}
$$

where $b_{2}(w, z)=-b-z_{2}^{2}-a z_{2}^{3}-\left(z_{2}^{3}-z_{2}^{1}+a z_{2}^{2}\right)\left(z_{2}^{2}-a z_{2}^{1}\right)$, which is flat with $\left(w_{1}^{1}, w_{2}^{1}\right)=\left(x_{\ell}, z_{\ell}\right)$ being a flat output. This system in the original coordinates is:

$$
\left(C h_{c}\right): \begin{cases}\dot{x}_{\ell}=\sigma\left(y_{\ell}-x_{\ell}\right) & \dot{x}_{r}=-y_{r}-z_{r} \\ \dot{y}_{\ell}=-k_{d} x_{\ell} z_{\ell}+r x_{\ell}-y_{\ell}+\frac{1}{\sigma} u_{1} & \dot{y}_{r}=x_{r}+a y_{r} \\ \dot{z}_{\ell}=k_{d} x_{\ell} y_{\ell}-\beta z_{\ell}+y_{r} u_{1} & \dot{z}_{r}=b+z_{r}\left(x_{r}-c\right)-u_{2} .\end{cases}
$$

The receiver knows completely the dynamics of the flat control system $\left(C h_{c}\right)$ as well as the transmitted output $\left(y_{1}(t), y_{2}(t)\right)=\left(x_{\ell}(t), z_{\ell}(t)\right)$ and therefore using the flatness property the original messages $u=\left(u_{1}(t), u_{2}(t)\right)$ can be recovered by the receiver as functions of the received output and its successive time-derivatives: $u_{1}(t)=\delta_{1}\left(y_{1}, \dot{y}_{1}, y_{2}\right)$ and $u_{2}(t)=$ $\delta_{1}\left(y_{1}, \ldots, y_{1}^{(5)}, y_{2}, \ldots, y_{2}^{(4)}\right)$.

Remark 5.1. An interesting question is why the unobserved part $z$ is needed. The unobserved $z$-subsystem plays an important role for increasing the safety. Indeed, represent the system $\left(C h_{c}\right)$ in $(w, z)$-coordinates, as NF1 given by (27), and notice that the message $u_{1}$ has a degree of security significantly lower than that of $u_{2}$. The $z$-subsystem can be seen as a second level of security. This motivates even more the use of quasi-indices: for a general two-output system, in order to decode $u_{1}$, we may need only the output $h_{1}=w_{1}^{1}$ (if the function $a_{1}$ of equation $\dot{w}_{1}^{\rho_{1}}=a_{1}(w)+u_{1}$, allowing to decode $u_{1}$, depends on $w_{1}^{j}$-variables only) 
but we never need the linking term $z_{2}^{1}$, however, we always need it for decoding $u_{2}$. Notice that for the proposed transmitter $\left(C h_{c}\right)$, the function $a_{1}$ involves both outputs $h_{1}=w_{1}^{1}$ and $h_{2}=w_{2}^{1}$. Another issue (closely related to the above question) is the motivation for using a two-output system for transmitting the two messages $u_{1}$ and $u_{2}$ (whose encodings are thus coupled), instead of using two independent systems, each one being used to encode a single message. Again it is clear that the proposed transmitter guarantees a higher security level for $u_{2}$ : in order to decode it, we have to decode beforehand $u_{1}$.

\subsection{Simulations}

In this section we present a preliminary analysis that first shows, using numerical simulations, that the chaotic behavior of the transmitter $(C h)$ is preserved by adding control vector fields multiplied by suitable periodic messages into the dynamics, and second, that for suitable amplitudes of the encoded messages, the messages are recovered with a good accuracy by the receiver. The amplitudes should not be too small, otherwise the messages will be hidden into the chaos or will have the same order of magnitudes as numerical errors or perturbations and it will be difficult to distinguish between them; they should not be too large either, otherwise the chaotic behavior will be strongly modified and the messages will be easily detected by a hacker. We will first give the phase plots for the uncontrolled dynamical transmitter composed of the Lorenz and Rössler circuits which are well known to be chaotic. Then we apply two sinusoidal input signals $u_{1}$ and $u_{2}$. We present three cases for different amplitudes of the inputs (the frequencies of $u_{1}$ and $u_{2}$ are the same for all cases). As expected, we observe that the larger the amplitudes of $u_{1}$ and $u_{2}$ are, the more the chaotic behavior is modified (but in all cases it seems to be preserved). A theoretical confirmation of this preliminary study will be the subject of a future work. Neither noise nor parameter uncertainties between the sender and the receiver nor the robustness with respect to different classical attacks have been considered here. It is, however, obvious that in practice those problems cannot be neglected and they have to be discussed in practical applications of the proposed method.

Figures $3 \mathrm{a}$ and $4 \mathrm{a}$ give the phase plots for the original Lorenz and Rössler subsystems (with no control), see the last paragraph of this section for the parameters values used in simulations. Figures $3 \mathrm{~b}$ and $4 \mathrm{~b}$ correspond to the solutions of the control system $\left(C h_{c}\right)$ and show that the chaotic behavior is preserved after modifying the original dynamical chaotic system $(C h)$ by applying the following inputs: $u_{1}=0.5+0.1 \sin (2 \pi t)$ and $u_{2}=0.5 \sin (1.8 \pi t)$ to the control system $\left(C h_{c}\right)$. For such (small) amplitudes, the first message is recovered with a good accuracy (Figure 5a), but the second one does not converge to the transmitted $u_{2}$ (Figure 5b). This is due to the fact that in order to decode $u_{2}$, we need the third derivative of $y_{r}$ (the linking term between the two chaotic subsystems) and even if the decoded $y_{r}$ is close to the transmitted one (Figure 5c), it still contains some approximation errors (namely, the pick around $3.5 \mathrm{~s}$ in Figure $5 \mathrm{c}$ ) that are amplified at each new differentiation and lead to a decoded $u_{2}$ which is very far from the transmitted one.

Figures $3 \mathrm{c}$ and $4 \mathrm{c}$ correspond to the solutions of the control system $\left(C h_{c}\right)$ obtained for $u_{1}=5+\sin (2 \pi t)$ and $u_{2}=5 \sin (1.8 \pi t)$. Notice that, the amplitude of the control values equals ten times that used in the previous example. For such inputs values, only the Lorenz strange attractor is slightly modified (a peak appears). Figures $6 \mathrm{a}$ and $6 \mathrm{~b}$ show that, for $u_{1}=5+\sin (2 \pi t)$ and $u_{2}=5 \sin (1.8 \pi t)$, the input $u_{1}$ (which corresponds to one of the confidential messages) can be exactly recovered after a very short time $(t \cong 0.1 \mathrm{~s})$ and $u_{2}$ can be recovered with a very good accuracy after $2 \mathrm{~s}$. In order to obtain $u_{2}$, first we have to 


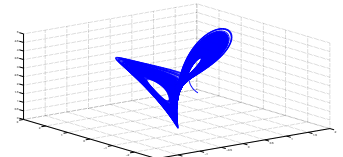

(a) No control.

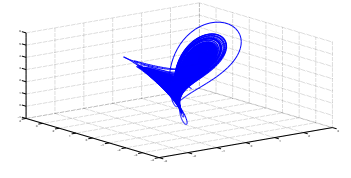

(b) Small amplitudes.

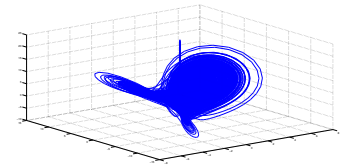

(c) Intermediate ampli- (d) Large amplitudes. tudes.

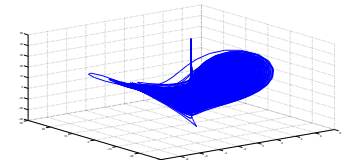

Figure 3: Phase plots for the Lorenz state variables for different inputs.

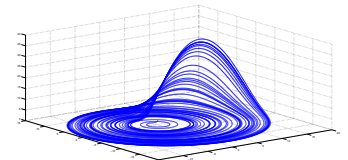

(a) No control.

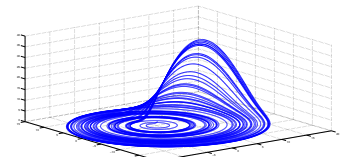

(b) Small amplitudes.
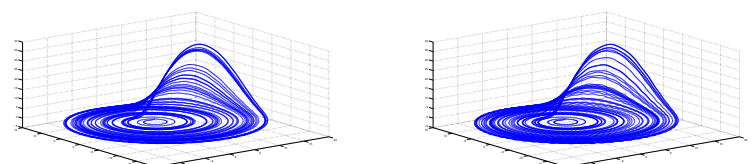

(c) Intermediate ampli- (d) Large amplitudes. tudes.

Figure 4: Phase plots for the Rössler state variables for different inputs.

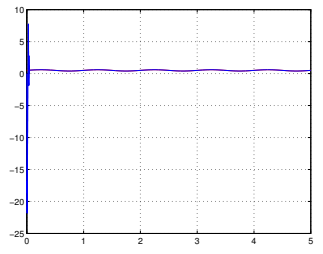

(a) $u_{1}$

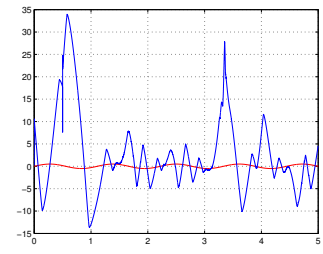

(b) $u_{2}$

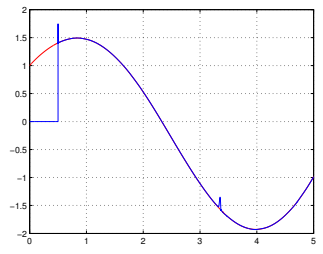

(c) $y_{r}$

Figure 5: To-be-sent messages and $y_{r}$ of the transmitter in red, decoded messages and decoded $y_{r}$ in blue for $u_{1}=0.5+0.1 \sin (2 \pi t), u_{2}=0.5 \sin (1.8 \pi t)$. 


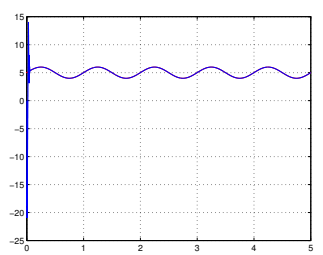

(a) $u_{1}$

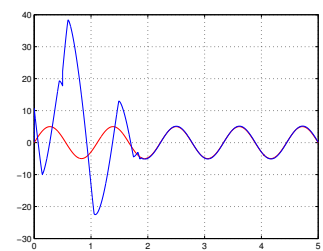

(b) $u_{2}$

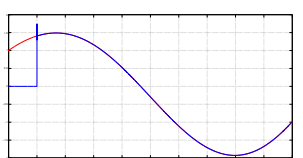

(c) $y_{r}$

Figure 6: To-be-sent messages and $y_{r}$ of the transmitter in red, decoded messages and decoded $y_{r}$ in blue for $u_{1}=5+\sin (2 \pi t), u_{2}=5 \sin (1.8 \pi t)$.

decode $u_{1}$, then $y_{r}$ and, finally, to differentiate three times the filtered $y_{r}$. Figure 6c shows that, after $0.5 \mathrm{~s}$, the real $y_{r}$ of the transmitter and the decoded one coincide. The purpose of this paper is not to present the receiver (observer or/and differentiator), nevertheless it is important to note that the presented simulations have been obtained using two homogeneous differentiators [16] in order to compute $u_{1}$ and $y_{r}$. Then, after filtering the obtained $y_{r}$, via a low pass filter, we use again a homogeneous differentiator to decode $u_{2}$. The above time constants $(0.1 \mathrm{~s}, 2 \mathrm{~s}$, etc. $)$ are related to the differentiators gains.

From Figures $3 \mathrm{~d}, 4 \mathrm{~d}$, we see that for $u_{1}=10+2 \sin (2 \pi t), u_{2}=10 \sin (1.8 \pi t)$, the chaotic behavior of the Lorenz and Rössler subsystems are modified. For such amplitudes, the messages are perfectly recovered by the receiver (as above, after $0.1 \mathrm{~s}$ and $2 \mathrm{~s}$, resp.) but the structure of the strange attractors are too strongly modified and a hacker would be able to detect the messages. We do not give the curves for the decoded messages since they are very similar to those of the previous case.

The parameters for the simulations are given by $\beta=8 / 3, \sigma=10, r=28, k_{d}=10$, $a=0.15, b=0.20, c=10$, with initial conditions $x_{\ell}(0)=y_{\ell}(0)=z_{\ell}(0)=1$ and $x_{r}(0)=$ $y_{r}(0)=z_{r}(0)=1$.

\section{Proofs}

\subsection{Proof of Theorem 3.1}

Proof of $(\mathcal{O} 1) \Rightarrow\left(\mathcal{O}_{2}\right)$. Consider the system $\Sigma$, around $x_{0}$, together with its output $h=$ $\left(h_{1}(x), \ldots, h_{m}(x)\right)$ and assume that there exist OQI's $\left(\rho_{1}, \ldots, \rho_{m}\right)$ at $x_{0}$. Around $x_{0}$, the system $\Sigma$ can be locally brought into $\dot{w}_{i}^{j}=w_{i}^{j+1}, 1 \leq j \leq \rho_{i}-1, \dot{w}_{i}^{\rho_{i}}=a_{i}(w), 1 \leq i \leq m$, with $w_{i}^{j}=L_{f}^{j-1} h_{i}$, for $1 \leq i \leq m$ and $1 \leq j \leq \rho_{i}$. For this form, define $g_{i}(w)=\frac{\partial}{\partial w_{i}^{\rho_{i}}}$, $1 \leq i \leq m$. It is clear that

$$
\Sigma_{c}:\left\{\begin{array}{l}
\dot{w}_{i}^{j}=w_{i}^{j+1}, \quad 1 \leq j \leq \rho_{i}-1, \\
\dot{w}_{i}^{\rho_{i}}=a_{i}(w)+u_{i}, \quad 1 \leq i \leq m,
\end{array}\right.
$$

is static feedback linearizable around $x_{0}$ (with linearizing outputs $h_{i}=w_{i}^{1}, 1 \leq i \leq m$ ) and thus $x$-flat at $x_{0}$ of differential weight $n+m$, with $h$ a minimal flat output.

Proof of $\left(\mathcal{O}_{2}\right) \Rightarrow(\mathcal{O} 1)$. Suppose that there exist $g_{1}, \ldots, g_{m}$, around $x_{0}$, such that the associated control system $\Sigma_{c}: \dot{x}=f(x)+\sum_{i=1}^{m} g_{i}(x) u_{i}$ is $x$-flat at $x_{0}$ of differential weight $n+m$, with $h=\left(h_{1}(x), \ldots, h_{m}(x)\right)$ being a minimal flat output. Let $\nu_{1}, \ldots, \nu_{m}$ denote the relative degrees of $h_{1}(x), \ldots, h_{m}(x)$, that is, $h_{i}^{\left(\nu_{i}\right)}$ is the lowest time-derivative of $h_{i}(x(t))$ depending explicitly on $u$. Since the flat outputs and all their successive derivatives are 
independent at $x_{0}$, we can introduce the new coordinates $\tilde{x}_{i}^{j}=L_{f}^{j-1} h_{i}$, for $1 \leq i \leq m$ and $1 \leq j \leq \nu_{i}$. We get

$$
\begin{aligned}
& \dot{\tilde{x}}_{i}^{j}=\tilde{x}_{i}^{j+1}, \quad 1 \leq j \leq \nu_{i}-1, \\
& \dot{\tilde{x}}_{i}^{\nu_{i}}=L_{f}^{\nu_{i}} h_{i}+\sum_{j=1}^{m}\left(L_{g_{j}} L_{f}^{\nu_{i}-1} h_{i}\right) u_{j}, \quad 1 \leq i \leq m .
\end{aligned}
$$

Flatness of differential weight $n+m$ implies that we necessarily have $\sum_{i=1}^{m} \nu_{i}=n$ and that the decoupling matrix $D_{i j}(x)=L_{g_{j}} L_{f}^{\nu_{i}-1} h_{i}(x)$, for $1 \leq i, j \leq m$, is of full rank around $x_{0}$ (otherwise the system would not be flat of differential weight $n+m$ ). It is clear that for the initial $\Sigma$, the integers $\rho_{i}=\nu_{i}, 1 \leq i \leq m$, are OQI's at $x_{0}$.

In order to complete the proof, observe that, by Lemma 4.1, $\operatorname{dim} \mathcal{H}(x)=n$, for any $x \in \mathbb{R}^{n}$, implies that OQI's exist on an open and dense subset $\mathcal{X}^{O} \subset \mathbb{R}^{n}$.

\subsection{Proof of Proposition 3.1}

Suppose that $(\Sigma, h)$ admits OQI's $\left(\rho_{1}, \ldots, \rho_{m}\right)$ at $x_{0}$. It follows from the proof of the implication $\left(\mathcal{O}_{2}\right) \Rightarrow(\mathcal{O} 1)$ of Theorem 3.1 that desired vector fields $g_{1}, \ldots, g_{m}$ satisfy (i) for a suitable invertible matrix $\left(D_{i j}(x)\right)$. Conversely, consider $g_{1}, \ldots, g_{m}$ satisfying (i). If $D(x)=I_{m}$, where $I_{m}$ denotes the identity $m \times m$-matrix, then by the proof of the implication $(\mathcal{O} 1) \Rightarrow(\mathcal{O} 2)$, we get $g_{i}=\frac{\partial}{\partial w_{i}^{\rho_{i}}}$. If we take $D(x)$ instead of $I_{m}$, then we have to replace $g(x) \mapsto g(x) \beta(x)$, where $\beta(x)=(D(x))^{-1}$ proving that for both choices of $g_{i}$ 's we get a feedback linearizable system for which $h$ is a flat output of differential weight $n+m$. Independently of $D(x)$, all corresponding vector fields $g_{1}, \ldots, g_{m}$ define the same distribution $\mathcal{G}$ given by (ii).

\subsection{Proof of Corollary 3.2}

Assume that $\left(\Sigma_{c}, h\right)$ is static feedback linearizable around $x_{0}$. Then by Theorem 3.1, there exists OQI's at $x_{0}$ and, in particular, $(\Sigma, h)$ is locally observable at $x_{0}$.

\subsection{Proof of Corollary 3.3}

Consider a linear dynamical system $\Sigma: \dot{x}=A x, y=C x$, and assume that $(A, C)$ is observable. Clearly, there exist OQI's for $(A, C)$ and vector fields $g_{1}, \ldots, g_{m}$ that render the system flat can be constructed with the help of condition Proposition 3.1 (i), in which we take $D_{i j}(x)=\delta_{i j}$. It is clear that $g_{i}=b_{i}, 1 \leq i \leq m$. Let us now suppose that there exist constant vector fields $g_{1}=b_{1}, \ldots, g_{m}=b_{m}$ such that the associated linear control system is flat at $x_{0}$. Since a flat control system is observable with respect to its flat output and since the observability of linear control systems does not depend on the control vector fields, it follows that $(A, C)$ is necessarily observable.

\subsection{Proof of Lemma 4.1}

For $1 \leq j \leq n$, denote $\mathscr{H}^{j}(x)=\operatorname{span}\left\{\mathrm{d} L_{f}^{q-1} h_{i}(x), 1 \leq i \leq m, 1 \leq q \leq j\right\}$ not to be confused with $\mathcal{H}^{j}$ of Section 4.1.1. The rank of each distribution $\mathscr{H}^{j}(x)$, for $1 \leq j \leq n$, is locally constant on an open and dense subset $\mathscr{X}^{j}$ of $\mathbb{R}^{n}$. So all those ranks are locally constant on $\mathcal{X}^{P}=\cap_{1 \leq j \leq n} \mathscr{X}^{j}$, open and dense subset of $\mathbb{R}^{n}$. On each connected component $X_{c}^{P}$ of $\mathcal{X}^{P}$, we have $\operatorname{dim} \mathscr{H}^{n}(x)=k \leq n$ (with $k$ that may depend on the component). It follows that on each $X_{c}^{P}$ there exist integers $\left(\rho_{1}, \ldots, \rho_{m}\right)$ such that $\sum_{i=1}^{m} \rho_{i}=k$ and 
$\mathscr{H}^{j}(x)=\operatorname{span}\left\{\mathrm{d} L_{f}^{q-1} h_{i}(x), 1 \leq i \leq m, 1 \leq q \leq \min \left\{j, \rho_{i}\right\}\right\}$ and thus on each component $X_{c}^{P}$ such that $k<n$ there exist POQI's (that may depend on the component) at any $x_{0} \in X_{c}^{P}$, and on each component $X_{c}^{P}$ such that $k=n$ (in which case we denote $X_{c}^{P}$ by $X_{c}^{O}$ ) there exist OQI's (that may depend on the component) at any $x_{0} \in X_{c}^{O}$. In the analytic case $k$ and $\left(\rho_{1}, \ldots, \rho_{m}\right)$ are the same for all components. The proof of Lemma 4.1 provides a construction of one choice of POQI's only: namely, the smallest $\left(\rho_{1}, \ldots, \rho_{m}\right)$ satisfying $\rho_{1} \geq \rho_{2} \geq \ldots \geq \rho_{m}$.

\subsection{Proof of Theorem 4.1}

Statement (Flat 2) follows directly from Theorem 3.1 while that of (Flat 3) from Theorems 4.2 and 4.4. In the observable case, by Lemma 4.1. OQI's exist on an open and dense subset of $\mathbb{R}^{n}$, while in the unobservable case, by the algorithm of Section 4.1.1. COQI's exist on an open and dense subset of $\mathbb{R}^{n}$, implying the first part of (Flat 1 ). Construction of $g_{1}, \ldots, g_{m}$, via differentiations and algebraic operations, is guaranteed by Proposition 3.1 and Corollary 3.1 (observable case) and Proposition 4.1 and Corollary 4.1 (unobservable case).

\subsection{Proof of Theorem 4.2}

Proof of (A1). The proof is contained in the algorithm of Section 4.1.1 which constructs, around any point of an open and dense subset $\mathcal{X}^{C}=\mathcal{X}^{\ell}$ of $\mathbb{R}^{n}$, dummy outputs $\psi^{1}, \ldots, \psi^{\ell}$ leading to COQI's at that point.

Proof of (A2). The algorithm of Section 4.1.1 constructs the vector field $g_{1}, \ldots g_{m}$ leading to the flat control system $(\mathrm{NF} 1, h)$. To show that, $h$ is indeed a flat output, notice that $w_{i}^{j}=h_{i}^{(j-1)}$, for $1 \leq i \leq m, 1 \leq j \leq \rho_{i}, h_{1}^{\left(\rho_{1}\right)}=\dot{w}_{1}^{\rho_{1}}=a_{1}(w)+u_{1}$ yielding $u_{1}=\delta_{1}\left(\bar{h}_{1}^{\rho_{1}}, \bar{h}_{2}^{\rho_{2}-1}, \ldots, \bar{h}_{m}^{\rho_{m}-1}\right)$, where $\bar{h}_{i}^{j}$ denotes $\bar{h}_{i}^{j}=\left(h_{i}, \dot{h}_{i}, \ldots, h_{i}^{(j)}\right)$. Then, provided that $u_{1} \neq 0$, equations $h_{i}^{\left(\rho_{i}\right)}=\dot{w}_{i}^{\rho_{i}}=a_{i}(w)+z_{i}^{1,1} u_{1}$, for $2 \leq i \leq m$, allow to express $z_{i}^{1,1}=$ $\gamma_{i}^{1,1}\left(\bar{h}_{1}^{\rho_{1}}, \bar{h}_{2}^{\rho_{2}}, \ldots, \bar{h}_{m}^{\rho_{m}}\right)$. By differentiating $z_{i}^{1,1}$, we get $z_{i}^{1,2}=\dot{z}_{i}^{1,1}=\gamma_{i}^{1,2}\left(\bar{h}_{1}^{\rho_{1}+1}, \bar{h}_{2}^{\rho_{2}+1}, \ldots, \bar{h}_{m}^{\rho_{m}+1}\right)$, for $2 \leq i \leq m$, and so on. For $2 \leq i \leq \bar{m}$, denote $\mu_{i}=\nu_{i}^{1}+\cdots+\nu_{i}^{\bar{\ell}}$ and $\mu=\max _{2 \leq i \leq \bar{m}}\left\{\mu_{i}\right\}$. In order to see that the differential weight of $h$ is at least $n+m+\mu$, recall that we have just expressed $u_{1}$ as $u_{1}=\delta_{1}\left(\bar{h}_{1}^{\rho_{1}}, \bar{h}_{2}^{\rho_{2}-1}, \ldots, \bar{h}_{m}^{\rho_{m}-1}\right)$ and the remaining $m-1$ inputs $u_{2}, \ldots, u_{m}$ appear at the bottom level of each $z_{i}$-chain. It follows that, in the best case, $u_{1}$ is the only input that needs to be differentiated in order to express all states and controls with the help the $h_{i}$ 's and their derivatives. For each $z_{i}$-chain, the input $u_{1}$ needs to be differentiated $\mu_{i}$ times. Hence, in the best case, the differential weight of $h$ is $n+m+\mu$. In some cases, we may need additionally to differentiate other inputs among $u_{2}, \ldots, u_{m}$. To understand this, consider the following simple example with $m=3, \ell=2, \nu_{2}^{1}=\nu_{3}^{1}=\nu_{2}^{2}=1$ and $\nu_{3}^{2}=3$, and for which the equations of the $z_{2^{-}}$and $z_{3}$-chains are:

$$
\begin{aligned}
\dot{z}_{2}^{1,1}=w_{1}^{1}+z_{2}^{2,1} u_{1} & \dot{z}_{3}^{1,1}=z_{2}^{1,1}+z_{3}^{2,1} u_{1} \\
\dot{z}_{2}^{2,1}=u_{2} & \dot{z}_{3}^{2, q}=\dot{z}_{3}^{2, q+1}, \quad q=1,2 \\
\dot{z}_{3}^{2,3} & =u_{3} .
\end{aligned}
$$

For this example, in addition to $u_{1}$, also the control $u_{2}$ necessarily has to be differentiated in order to express all $z_{3}^{s, q}$-states and control $u_{3}$ in terms of $h$ and its derivatives. In follows that, in general, the differential weight of $h$ is greater than $n+m+\mu$. 


\subsection{Proof of Theorem 4.3}

Proof of (B1). The proof is given by the algorithm constructing the flat control system NF2 in Section 4.1.2.

Proof of (B2). Consider the system $\Sigma$, around $x_{0}$, together with its output $h=\left(h_{1}(x), \ldots, h_{m}(x)\right)$ and suppose that there exist $g_{1}, \ldots, g_{m}$ such that the associated control system $\Sigma_{c}: \dot{x}=$ $f(x)+\sum_{i=1}^{m} g_{i}(x) u_{i}$ is $x$-flat at $x_{0}$ with $\varphi=\left(h_{1}(x), \ldots, h_{m}(x)\right)$ an $x$-flat output, defined in a neighborhood of $x_{0}$. Recall that $n-k=p(m-1)+r$ is the Euclidean division of the observability defect $n-k$ and $m-1$. We will show that the differential weight of $\varphi=\left(h_{1}(x), \ldots, h_{m}(x)\right)$ is at least $n+m+p^{*}$ (where $p^{*}=p$, if $r=0$ and $p^{*}=p+1$ otherwise). We denote by $s_{i}$ the order of the highest derivative of $\varphi_{i}=h_{i}$, for $1 \leq i \leq m$, involved in the expression of $x$ and $u$, i.e.,

$$
x=\gamma\left(\bar{\varphi}_{1}^{s_{1}}, \ldots, \bar{\varphi}_{m}^{s_{m}}\right) \text { and } u=\delta\left(\bar{\varphi}_{1}^{s_{1}}, \ldots, \bar{\varphi}_{m}^{s_{m}}\right),
$$

where $\bar{\varphi}_{i}^{j}=\left(\varphi_{i}, \dot{\varphi}_{i}, \ldots, \varphi_{i}^{(j)}\right)$ and the differential weight of $\varphi=h$ equals $\sum_{i=1}^{m}\left(s_{i}+1\right)$.

Let $\nu_{i}$, for $1 \leq i \leq m$, be the relative degree of $\varphi_{i}=h_{i}$, that is, the smallest integer such that the derivative $h_{i}^{\left(\nu_{i}\right)}$ depends explicitly on the control $u$. We clearly have $\nu_{i} \geq 1$ (since all $h_{i}$ depend on $x$ only), the functions $L_{f}^{j-1} \varphi_{i}=L_{f}^{j-1} h_{i}$, for $1 \leq i \leq m$ and $1 \leq j \leq \nu_{i}$, are independent (as successive derivatives of flat outputs) and $\mathrm{d} L_{f}^{j-1} h_{i} \in \mathcal{H}$, so $\operatorname{dim} \mathcal{H}(x)=k$ implies $\sum_{i=1}^{m} \nu_{i} \leq k$ (and, in particular, $\sum_{i=1}^{m} \nu_{i}<n$, since $k<n$ ). For $1 \leq i, j \leq m$, denote rk $\left(L_{g_{j}} L_{f}^{\nu_{i}-1} h_{i}\right)\left(x_{0}\right)=\mathrm{rk}\left(D_{i j}\right)\left(x_{0}\right)=d>0$. We clearly have $d \leq m-1$, otherwise the control system $\Sigma_{c}$ would not be flat since $\sum_{i=1}^{m} \nu_{i}<n$. We first suppose that the rank $d$ of the decoupling matrix $\left(D_{i j}(x)\right)$ is constant around $x_{0}$. By the definition of the relative degree, we introduce new coordinates $\tilde{w}_{i}^{j}=L_{f}^{j-1} \varphi_{i}=L_{f}^{j-1} h_{i}$, for $1 \leq i \leq m$ and $1 \leq j \leq \nu_{i}$, and let $\xi$ be any coordinates completing the $\tilde{w}$-variables to a coordinate system and thus $\operatorname{dim} \xi \geq n-k$ (we use $\tilde{w}$, and not $w$ because, in general $\nu_{i} \neq \rho_{i}$, where $\rho_{i}$ 's are POQI's of $(\Sigma, h))$. By applying a suitable feedback transformation (permute the outputs $h_{i}$, if necessary), the system in the $(\tilde{w}, \xi)$-coordinates reads

$$
\begin{aligned}
\dot{\tilde{w}}_{i}^{j}=\tilde{w}_{i}^{j+1}, 1 \leq j \leq \nu_{i}-1, & \dot{\tilde{w}}_{i}^{j}=\tilde{w}_{i}^{j+1}, 1 \leq j \leq \nu_{i}-1, \\
\dot{\tilde{w}}_{i}^{\nu_{i}}=v_{i}, 1 \leq i \leq d, & \dot{\tilde{w}}_{i}^{\nu_{i}}=a_{i}\left(\tilde{w}, \xi, v_{1}, \ldots, v_{d}\right), \quad d+1 \leq i \leq m, \\
& \dot{\xi}=G(\tilde{w}, \xi, v),
\end{aligned}
$$

for a smooth map $G$ and smooth functions $a_{i}$, all of them affine with respect to the controls. By assumption, the above system is flat with $\varphi=\left(\tilde{w}_{1}^{1}, \ldots, \tilde{w}_{m}^{1}\right)$ a flat output. We have $\tilde{w}_{i}^{j}=h_{i}^{(j-1)}$, for $1 \leq i \leq m, 1 \leq j \leq \nu_{i}$, and $v_{i}=h_{i}^{\left(\nu_{i}\right)}$, for $1 \leq i \leq d$. Thus the remaining $n-\sum_{i=1}^{m} \nu_{i}$ states $\xi_{i}$ and remaining $m-d$ controls $v_{d+1}, \ldots, v_{m}$ are expressed via $h_{i}^{\left(\nu_{i}+j\right)}$, for $d+1 \leq i \leq m$ and $0 \leq j \leq s_{i}-\nu_{i}$. This is possible only if $\sum_{i=d+1}^{m}\left(s_{i}-\nu_{i}+1\right) \geq \operatorname{dim} \xi+m-d$ implying

$$
\sum_{i=d+1}^{m}\left(s_{i}-\nu_{i}\right) \geq \operatorname{dim} \xi \geq n-k=p(m-1)+r .
$$

We claim that there exits $s_{i^{*}}-\nu_{i^{*}} \geq p^{*}$; if not, then $s_{i}-\nu_{i}<p^{*}$, for all $d+1 \leq i \leq m$, and in the case $r=0$ (implying $p^{*}=p$ ), we have $\sum_{i=d+1}^{m}\left(s_{i}-\nu_{i}\right)<p(m-d) \leq p(m-1)$, while in the case $r \neq 0$ (implying $\left.p^{*}=p+1\right)$, we have $\sum_{i=d+1}^{m}\left(s_{i}-\nu_{i}\right) \leq p(m-d) \leq p(m-1)<p(m-1)+r$, which, in both cases, contradicts $(29)$. Each $h_{i}^{\left(s_{i}\right)}$ depends on $s_{i}-\nu_{i}$ derivatives of at least one control among $v_{d+1}, \ldots, v_{m}$, so $h_{i^{*}}^{\left(s_{i^{*}}\right)}$ depends on at least $p^{*}$ of them and the differential weight of $\varphi=\left(h_{1}(x), \ldots, h_{m}(x)\right)$ is at least $n+m+p^{*}$ around $x_{0}$. The set of points around which the decoupling matrix $\left(D_{i j}(x)\right)$ is of constant rank is open and dense in $\mathbb{R}^{n}$, so the differential weight of $\varphi=\left(h_{1}(x), \ldots, h_{m}(x)\right)$ is at least $n+m+p^{*}$ everywhere. 


\subsection{Proof of Theorem 4.4}

Proof of (A1)'. By Lemma 4.1. $(\Sigma, h)$ admits POQI's $\left(\rho_{1}, \ldots, \rho_{m}\right)$ on an open and dense subset $\mathcal{X}^{P}$ of $\mathbb{R}^{n}$. Consider $(\Sigma, h)$ around any point $x_{0} \in \mathcal{X}^{P}$ and choose any $z$-coordinates $\left(z_{m^{\prime}+1}^{1}, \ldots, z_{m}^{1}\right)$, where $m^{\prime}=m-r$, completing, around $x_{0}$, the $w$-coordinates, given by 10 , to a local coordinate system (recall that $n-k=r<m-1$ ). Put $\psi_{i}^{1}=z_{i}^{1}$, for $m^{\prime}+1 \leq i \leq m$. It is clear that the $r$-tuple of dummy outputs $\psi^{1}=\left(\psi_{m^{\prime}+1}^{1}, \ldots, \psi_{m}^{1}\right)$ completes the original $h$ to a new output $h^{1}=\left(h, \psi^{1}\right)$ such that $\left(\rho_{1}, \ldots, \rho_{m}, 1, \ldots, 1\right)$ are OQI's of $\left(\Sigma, h^{1}\right)$ at $x_{0}$, so they also form COQI's of $(\Sigma, h)$ at $x_{0}$, see Definition 4.2 and the remark following it.

Proof of (A2)'. The proof is given by the (constructive) algorithm providing the construction of the flat control system NF3 associated to $(\Sigma, h)$ in Section 4.2.1.

Proof of (B2)'. Repeat the proof of item (B2) of Theorem 4.3 for $p=0$ and $r \neq 0$.

\section{Acknowledgment}

We would like to thank anonymous reviewers whose suggestions and remarks have substantially improved the final presentation of the paper.

\section{References}

[1] J. Barbot, M. Fliess, And T. Floquet, An algebraic framework for the design of nonlinear observers with unknown inputs, in Proc. IEEE CDC, 2007, pp. 384-389.

[2] S. Bingulac And R. KRTolica, On admissibility of pseudoobservability and pseudocontrollability indexes, IEEE Trans. Automat. Control, 32 (1987), pp. 920-922.

[3] B. Dravie, P. Guillot, And G. Millérioux, Flatness and structural analysis as a constructive framework for private communication, Nonlinear Analysis: Hybrid Systems, 30 (2018), pp. 92-105.

[4] M. Fliess, J. Lévine, P. Martin, and P. Rouchon, Flatness and defect of nonlinear systems: introductory theory and examples, Internat. J. Control, 61 (1995), pp. $1327-1361$.

[5] M. Fliess, J. Lévine, P. Martin, And P. Rouchon, A Lie-Bäcklund approach equivalence and flatness of nonlinear systems, IEEE Trans. Automat. Control, 44 (1999), pp. 922-937.

[6] K. Fritzsche And K. RÖBEnACK, On the computation of differentially flat inputs, in Proc. of the 22nd ICSTCC, IEEE, 2018, pp. 12-19.

[7] J.-P. Gauthier, H. Hammouri, and S. Othman, A simple observer for nonlinear systems with applications to bioreactors, IEEE Trans. Automat. Control, 37 (1992), pp. $875-880$.

[8] K. Graichen, V. Hagenmeyer, And M. Zeitz, A new approach to inversion-based feedforward control design for nonlinear systems, Automatica, 41 (2005), pp. 2033 2041. 
[9] H. Hammouri and J. De LeOn Morales, Observer synthesis for state-affine systems, in Proc. IEEE CDC, vol. 2, IEEE, 1990, pp. 784-785.

[10] R. Hermann And A. J. Krener, Nonlinear controllability and observability, IEEE Trans. Automat. Control, 22 (1977), pp. 728-740.

[11] L. Hunt And R. Su, Linear equivalents of nonlinear time varying systems, in Proc. MTNS, Santa Monica, CA, 1981, pp. 119-123.

[12] A. Isidori, C. Moog, And A. De LuCA, A sufficient condition for full linearization via dynamic state feedback, in Proc. IEEE CDC, vol. 25, IEEE, 1986, pp. 203-208.

[13] B. JAKUBCZYK, Invariants of dynamic feedback and free systems, in Proc. ECC, 1993, pp. 1510-1513.

[14] B. Jakubczyk And W. Respondek, On linearization of control systems, Bull. Acad. Polonaise Sci. Ser. Sci. Math., (1980), pp. 517-522.

[15] A. KRener And W. Respondek, Nonlinear observers with linearizable error dynamics, SIAM J. Control Optim., 23 (1985), pp. 197-216.

[16] A. Levant, Homogeneity approach to high-order sliding mode design, Automatica, 41 (2005), pp. 823-830.

[17] J. LÉvine, Analysis and Control of Nonlinear Systems: A Flatness-Based Approach, Springer, 2009.

[18] E. N. LoREnz, Deterministic nonperiodic flow, Journal of the Atmospheric Sciences, 20 (1963), pp. 130-141.

[19] P. Martin, Contribution à l'étude des systèmes différentiellement plats, PhD thesis, l'Ecole Nationale Supérieure de Mines de Paris, 1992.

[20] P. Martin, P. Rouchon, And R. Murray, Flat systems, equivalence and trajectory generation, CDS Technical Report, Caltech, (2003).

[21] F. Nicolau And W. Respondek, Flatness of multi-input control-affine systems linearizable via one-fold prolongation, SIAM J. Control Optim., 55 (2017), pp. 3171-3203.

[22] F. Nicolau, W. Respondek, And J. Barbot, Constructing flat inputs for twooutput systems, in Proc. MTNS, 2018, pp. 414-421.

[23] F. Nicolau, W. Respondek, J. Barbot, And A. Ouslimani, Secure communication with the help of flat inputs for chaotic systems, IFAC-PapersOnLine, 51 (2018), pp. 109 - 114. 5th IFAC Conference on Analysis and Control of Chaotic Systems CHAOS 2018.

[24] H. NiJmeijer And I. MAREels, An observer looks at synchronization, IEEE Trans. Circuits Syst. I: Fundamental theory and Applications, 44 (1997), pp. 882-890.

[25] L. M. Pecora And T. L. Carroll, Synchronization in chaotic systems, Physical review letters, 64 (1990), pp. 1196-1199. 
[26] J. Pomet, A differential geometric setting for dynamic equivalence and dynamic linearization, Banach Center Publ., Vol. 32, (1995), pp. 319-339.

[27] W. Respondek, Right and left invertibility of nonlinear control systems, in Sussmann. Nonlinear controllability and optimal control, Routledge New York, 1990, pp. 133-176.

[28] W. RespondeK, Symmetries and minimal flat outputs of nonlinear control systems, in New Trends in Nonlinear Dynamics and Control and their Applications, vol. LNCIS 295, Springer, 2003, pp. 65-86.

[29] O. Rössler, An equation for continuous chaos, Phys. Let. A, 57 (1976), pp. 397-398.

[30] P. TAn, G. MilléRIOuX, And J. DAafouz, Invertibility, flatness and identifiability of switched linear dynamical systems: an application to secure communications, in 47th IEEE CDC'08, 2008, pp. 959-964.

[31] S. Waldherr And M. Zeitz, Conditions for the existence of a flat input, Internat. J. Control, 81 (2008), pp. 439-443.

[32] S. Waldherr And M. Zeitz, Flat inputs in the mimo case, IFAC Proceedings Volumes, 43 (2010), pp. 695-700.

[33] X. Xia And W. GaO, Non-linear observer design by observer canonical forms, Internat. J. Control, 47 (1988), pp. 1081-1100.

[34] X. XIA And W. GaO, Nonlinear observer design by observer error linearization, SIAM J. Control Optim., 27 (1989), pp. 199-216. 